\title{
Transport and Accretion in Planet-Forming Disks
}

\author{
Neal J. Turner \\ Jet Propulsion Laboratory, California Institute of Technology \\ Sébastien Fromang \\ Service d'Astrophysique de Saclay \\ Charles Gammie \\ University of Illinois at Urbana-Champaign \\ Hubert Klahr \\ Max Planck Institute for Astronomy \\ Geoffroy Lesur \\ Institute for Planetology and Astrophysics of Grenoble \\ Mark Wardle \\ Macquarie University \\ Xue-Ning Bai \\ Harvard-Smithsonian Center for Astrophysics
}

\begin{abstract}
Planets appear to form in environments shaped by the gas flowing through protostellar disks to the central young stars. The flows in turn are governed by orbital angular momentum transfer. In this chapter we summarize current understanding of the transfer processes best able to account for the flows, including magneto-rotational turbulence, magnetically-launched winds, self-gravitational instability and vortices driven by hydrodynamical instabilities. For each in turn we outline the major achievements of the past few years and the outstanding questions. We underscore the requirements for operation, especially ionization for the magnetic processes and heating and cooling for the others. We describe the distribution and strength of the resulting flows and compare with the long-used phenomenological $\alpha$-picture, highlighting issues where the fuller physical picture yields substantially different answers. We also discuss the links between magnetized turbulence and magnetically-launched outflows, and between magnetized turbulence and hydrodynamical vortices. We end with a summary of the status of efforts to detect specific signatures of the flows.
\end{abstract}

\section{Introduction}

A key to the evolution of the planet-forming material in protostellar disks is the angular momentum. The angular momentum per unit mass of gas orbiting just above the star's surface is only $1 \%$ that of gas near the disk's outer edge. Sustaining the accretion on the star therefore requires taking almost all the angular momentum out of the accreting matter. Since the angular momentum cannot be destroyed it must be handed off to other material. The gas receiving the angular momentum could move radially outward, or could join outflows escaping above and below the disk. The gas losing angular momentum and spiraling inward releases gravitational potential energy into turbulence, vortices or magnetic dissipation, which can stir and heat the flow.

Angular momentum transport processes that could be important under the right circumstances include magnetohydrodynamical turbulence initiated by the magneto-rotational instability, outflows, hydrodynamical processes and gravitational instability. In several of these the large-scale transport is built up from smallerscale gas motions such as turbulent eddies, spiral waves or long-lived vortices. In addition to governing the flow of gas to the star, these small-scale motions can control the mixing of gas molecules and dust grains along the radial and vertical gradients in temperature, density and radiation intensity (Fromang and Papaloizou. 2006: Ciesla and Cuzzi, 2006; Turner et al. | 2010; Semenov and Wiebe, 2011). The small-scale motions also can alter the growth of dust grains into bigger bodies 
(Brauer et al. 2008, Okuzumi, 2009: Zsom et al., 2010), the collisional evolution of planetesimals (Gressel et al. 2011) and the orbital migration of protoplanets ( Nelson and Papaloizou, 2004).

In this chapter we review what is known about the basic operation of these transport processes and when and in what regions they work. We restrict ourselves to processes originating in the disk. For example we do not consider either the influence of a companion star (Lin and Papaloizou, 1993), the shear between the disk surface and infalling material (Ruzmaikina, 1982) or the interaction of the disk with the young star's magnetosphere (Romanova et al. 2013). Furthermore we devote most attention to work published since the previous volume in this series.

We examine the magneto-rotational instability's linear growth (\$2) and development into saturated turbulence ( $\$ 3 ;$; disk-driven winds ( $\$ 4) ;$ and selfgravitational (\$5) and hydrodynamical instabilities (\$6). Finally we discuss the prospects for observing the signatures of each transport process (77) and the outlook for the next few years ( 88 ).

\section{The Linear Magneto-Rotational Instability}

Study of the linear magneto-rotational instability (MRI) is warranted by the desire to understand the mechanism driving magnetohydrodynamical (MHD) turbulence and the conditions under which driving occurs, so we can map the MRI-active regions in protoplanetary disks. The disks' weak ionization can lead to magnetic fluctuations being damped. While linear analysis does not address the non-linear saturated state, it provides a good check on numerical codes during the initial departure from equilibrium. Local linear analyses have also proven surprisingly good at pinpointing the boundaries of the MHD turbulent regions in stratified simulations.

We first review the behavior of the MRI in ideal MHD and then describe the different behaviors in the presence of Ohmic and ambipolar diffusion and Hall drift. We conclude with a discussion of the ionization equilibrium and the implications for magnetic activity.

\subsection{MRI Under Ideal MHD}

MRI in ideal MHD Couette flows was discovered in the late 1950s Chandrasekhar, 1960; Velikhov, 1959) but its astrophysical applications were appreciated only with rediscovery in the context of accretion disks (Balbus and Hawley, 1991). The basic mechanism relies on angular momentum transfer by magnetic tension coupling fluid elements at neighbouring radii. As the fluid elements drift apart under the orbital shear, magnetic tension transfers angular momentum from the inner fluid element to the outer one. The inner element moves inward and speeds up, the outer moves outward and slows down, increasing the rate at which the elements drift apart and leading to a runaway. The key is that the magnetic field is weak enough so magnetic tension cannot overcome the tendency of the fluid elements to separate due to the shear.

The result is a robust instability, relying only on a magnetic field embedded in a rotating shear flow in which angular momentum decreases outward. For a simple initially-vertical magnetic field, the most unstable mode is axisymmetric with growth rate $\frac{3}{4} \Omega \sqrt{B a l-}$ bus and Hawley, 1991) and vertical wavelength near $2 \pi v_{A z} / \Omega$. This rate has proven to be a general maximum in collisional plasmas under Keplerian rotation. The growth rate is reduced if the plasma beta parameter $\beta=P_{\text {gas }} / P_{\text {magnetic }}<1$, because the fastestgrowing mode no longer fits within the disk thickness. Adding a toroidal magnetic component such that $\beta<1$ reduces the fastest linear growth rate without much changing the wavelength. The growth rate approaches zero as $\beta \rightarrow 0$ if the field is within $30^{\circ}$ of toroidal (Kim and Ostriker, 2000). For strictly toroidal fields, the fastest modes' azimuthal wavelength again is near $2 \pi v_{A \phi} / \Omega$ but the vertical wavelength is vanishingly small (Terquem and Papaloizou, 1996). As with inclined fields, growth is slower if $\beta \lesssim 1$ Kim and $\mathrm{Os}$ triker, 2000). When the toroidal fields' $\beta \lesssim 10$, the Parker magnetic buoyancy instability grows faster than the MRI (Terquem and Papaloizou, 1996) and expels some of the magnetic energy into the disk atmosphere (Hirose and Turner, 2011).

\subsection{MRI Under Ohmic MHD}

The effect of Ohmic resistivity $\eta$ on the MRI is straightforward: it tends to erase magnetic fluctuations on length scales shorter than $\eta / v_{A}$. The important length scale for the MRI is the fastest growing wavelength $2 \pi v_{A} / \Omega$, so the dimensionless number that determines its effect on the MRI is the Elsasser number $\Lambda=v_{A}^{2} /(\eta \Omega)$. Local and global linear instabilities are damped when the time to diffuse across the fastestgrowing wavelength is less than the growth time, corresponding to $\Lambda<1$ (Jin, 1996, Sano and Miyama. 1999). This linear criterion is also a good estimator for the onset of turbulence (Sano and Inutsuka, 2001 Turner et al., 2007).

\subsection{MRI Under Ambipolar MHD}

MRI under ambipolar diffusion behaves similarly to the Ohmic case but there are some subtle differences because ambipolar diffusion does not dissipate fieldparallel currents. For strictly vertical initial fields, there are no field-parallel currents in the initial and perturbed states and the linear MRI behaves as in the Ohmic case, but with $\Lambda$ replaced by $A m=v_{A}^{2} /\left(\eta_{A} \Omega\right)$, where $\eta_{A}$ is the diffusivity due to ambipolar drift Blaes and Balbus, 1994, Wardle, 1999). 
This degeneracy is broken if the initial field has toroidal and vertical components. Then perturbations with a restricted range of wavevector orientations may be destabilised by ambipolar diffusion. This only partly compensates for the lower growth rate associated with the tilt in the field or with Ohmic resistivity if this is also present (Desch, 2004, Kunz and Balbus, 2004 ), and so appears to be of minor consequence.

\subsection{MRI Under Hall MHD}

Hall drift, characterised by transport coefficient $\eta_{H}$ and dimensionless number $\Lambda_{H}=v_{A}^{2} /\left(\eta_{H} \Omega\right)$, differs fundamentally from resistivity and ambipolar diffusion. It makes magnetic field lines drift through the gas in the direction of the current density, giving a tendency to twist; no dissipation is involved. Hall drift therefore permits the magnetic field to restructure in strange ways with no energy penalty. For example, Hall drift tends to rotate the MRI-induced buckling of an initially vertical field clockwise about the initial direction $\sqrt{\text { War- }}$ dle, 1999). If this is parallel to the rotation axis, Hall drift acts in concert with the Keplerian shear in generating toroidal from radial field. The maximum growth rate remains at $\frac{3}{4} \Omega$ but the most unstable wavelength becomes longer. If the initial field and rotation are antiparallel, Hall drift acts against the Keplerian shear, and completely suppresses the MRI if $\Lambda_{H}<0.5$.

Because Hall drift acts directly with or against the Keplerian shear in generating $B_{\phi}$ from $B_{r}$, it strongly modifies the linear MRI when $\Lambda_{H}<1$, irrespective of the magnitudes of $\Lambda$ and $A m$. For the "favorable" case $B_{z}>0$, if $\Lambda_{H}<\min (\Lambda, A m)$ the maximum growth rate is $\frac{3}{4} \Omega$ even if $\Lambda$ and/or $A m$ are small. Even if $\Lambda$ and/or $A m<\Lambda_{H}$, Hall drift mitigates the damping effects of Ohmic or ambipolar diffusion, increasing the effective Elsasser number by the factor $\Lambda_{H}^{-1 / 2}$ (Wardle, 1999, Wardle and Salmeron, 2012).

A toroidal component in the initial field introduces trigonometric corrections to the MRI growth rate and wavelength, and additional mitigation of Ohmic damping by ambipolar diffusion (Pandey and Wardle, 2012).

Hall drift is able to restructure the field with no associated dissipation. Its ability to mitigate or exacerbate damping of the MRI, and the fact that Ohmic and ambipolar diffusion and Hall drift all scale differently with the field strength $B$, may give rise to interesting behavior in the non-linear state.

\subsection{Ionization and Recombination}

The transport coefficients $\eta, \eta_{A}$, and $\eta_{H}$ depend on the abundances of the charged particles in the weakly ionized gas in protoplanetary disks. The ionization sources in protoplanetary disks are thermal collisions, stellar ultraviolet (UV) and X-ray photons, interstellar cosmic rays and the decay of radionuclides. Collisional ionization is important at $\gtrsim 1000 K$, tempera- tures found only within $0.1-1$ AU of the star depending on the mass flow rate, and also high in the disk atmosphere. Only the elements with the lowest ionization potentials are ionized, so the ionization fraction is capped at the gas-phase abundance of the most common alkali metals.

Far-UV continuum photons are absorbed in the disk's uppermost $0.001-0.1 \mathrm{~g} \mathrm{~cm}^{-2}$ Bergin et al. 2007. Perez-Becker and Chiang, 2011a) unless absorbed first by an intervening disk wind emerging from smaller radii (Ferro-Fontán and Gómez de Castro, 2003; Panoglou et al., 2012). Lyman- $\alpha$ photons propagate by resonant scattering and so may enhance ionization rates somewhat deeper in the disk (Bethell and Bergin, 2011).

Stellar X-rays are absorbed in the uppermost $10 \mathrm{~g} \mathrm{~cm}^{-2}$ (Igea and Glassgold, 1999 , Mohanty et al. 2013), yielding unattenuated ionization rates around $10^{-10} \mathrm{~s}^{-1}$ at $1 \mathrm{AU}$ for the median X-ray luminosity of $10^{30} \mathrm{erg} \mathrm{s}^{-1}$ measured among young Solar-mass stars in Orion (Garmire et al. 2000).

Interstellar cosmic rays are largely screened out by the young star's wind, according to an extrapolation of the Solar relation between spot coverage and cosmic ray modulation to young stars' greater spot covering fractions (Cleeves et al., 2013 ). On the other hand, if the stellar wind is restricted to the poles and the incoming cosmic rays interact mostly with a disk wind threaded by poloidal magnetic fields, the energetic particles are both focused and mirrored by the fields' pinching toward the equatorial plane. The first increases and the second decreases the ionization rate, on balance yielding only small changes compared with ambient conditions (Desch et al., 2004, Cleeves et al. 2013). If cosmic rays do reach the disk, then they will penetrate much deeper than X-rays, to $100 \mathrm{~g} \mathrm{~cm}^{-2}$ (Umebayashi and Nakano, 1981, 2009), but yield a lower unattenuated ionization rate of about $10^{-17} \mathrm{~s}^{-1}$.

The radionuclides ionize at lower rates, and the decay products are absorbed inside the solid material if contained in particles bigger than $\sim 1 \mathrm{~mm}$ Umebayashi et al. 2013). Most of the radionuclide ionization comes from ${ }^{26} \mathrm{Al}$, if present. Its half-life of $0.7 \mathrm{Myr}$ and initial abundance inferred from decay products in primitive meteorites yield an ionization rate $(7-10) \times 10^{-19} \mathrm{~s}^{-1}$ (Umebayashi and Nakano, 2009).

Clearly there is a great deal of variation in the overall ionization rate with distance from the star and depth in the disk.

The electrons and molecular ions created by ionizations may recombine directly, or by charge transfer to metal ions followed by radiative recombination (e.g. Umebayashi and Nakano, 1990, Ilgner and Nelson, 2006a). The latter rate is relatively low, making metal ions the dominant positive species in the gas phase except at the highest ionization levels. At low temperatures the metal atoms become adsorbed on 
grain surfaces, removing them from the picture. Dust grains play a major role through the competitive sticking of electrons and ions from the gas phase and subsequent recombination on the grains (Sano et al. 2000).

For a given grain population, the important parameter is $\zeta / n_{\mathrm{H}}$, the ratio of the ionization rate per $\mathrm{H}$ nucleus to the number of nuclei per unit volume. At high ionization levels most charge resides in the gas phase. Grains acquire a net negative charge because of the higher thermal speed of the electrons, but the fraction of negative charge held by grains is small. As an electron is more likely to encounter an ion in the gas phase than a grain, the majority of recombinations occur in the gas phase. At intermediate ionization levels, electrons are more likely to stick to a grain surface before encountering an ion in the gas phase. In this regime, most recombinations occur via the sticking of ions to negatively charged grains. At low ionization levels, most grains are uncharged, and ions and electrons tend to stick to neutral grains before recombining. Recombinations occur primarily via the collision of oppositely charged grains.

\subsection{Conductivity}

Ionization fractions are highest in the disk atmosphere, where the UV and X-ray photons are absorbed (in the upper 0.01 and $10 \mathrm{~g} \mathrm{~cm}^{-2}$, respectively), and low densities reduce the recombination rates. Deeper in the disk, the ionization levels plummet due to shielding by the overlying layers and the increase in recombination rates with density.

The overall ionization level and the relative abundances of ions, electrons and charged grains therefore vary strongly with depth, with concomitant changes to the Ohmic and ambipolar diffusivities and Hall drift. The relative importance of the non-ideal terms is determined by the ratio $n_{\mathrm{H}} / B$, which controls the degree to which neutral collisions decouple charged species from the magnetic field. At successively higher densities, first grains, then ions, and finally electrons are decoupled. Roughly speaking, ambipolar diffusion dominates at low densities on strong magnetic fields, when ions and electrons remain coupled. The Ohmic term typically dominates at the high densities near the midplane and on weak magnetic fields. The Hall term dominates over a broad range of intermediate conditions, when ions are decoupled from the fields by collisions with neutrals, but electrons are not Wardle, 2007: Salmeron and Wardle, 2008).

Ohmic diffusivity is always determined by the electron fraction even when electrons are not the most abundant charged particle. Ambipolar diffusivity is typically determined by the ion density, but can be set by the charged grains if these are small and abundant (Bai, 2011b); at low ionization levels charged grains again play a role. The Hall drift is more complex, as it is controlled by the mismatch in the degree to which neutral collisions decouple positive and negative species from the magnetic field. At low densities negatively charged grains are decoupled and ions and electrons are coupled, implying $\eta_{H}<0$. At intermediate densities ions decouple too and $\eta_{H}>0$.

\subsection{Active Layers and Dead Zone}

A simple local criterion for MRI turbulence is that the linear instability has an unstable mode with wavelength shorter than the disk scale height — i.e., that Ohmic and ambipolar diffusion are not too severe. Hall drift enters by modifying the twisting of the field in response to the Keplerian shear, and may aid or hinder the growth. By way of example, for vertical fields this criterion may be written

$$
\left(\frac{1}{\Lambda}+\frac{1}{A m}\right)^{2}<\left(2+\frac{s}{\Lambda_{H}}\right)\left(\frac{3 \beta}{16 \pi^{2}}-\frac{1}{2}-\frac{s}{\Lambda_{H}}\right)
$$

where $s=\operatorname{sign}\left(B_{z}\right)$ (Wardle and Salmeron, 2012, used $1 / k$ rather than $\lambda$ in the criterion $k h=1$, so in their equivalent expression $\beta$ has coefficient $\frac{3}{4}$ ). This criterion describes reasonably well whether turbulence develops in simulations in unstratified boxes in resistive MHD (Sano and Inutsuka, 2001), ambipolar diffusion (Hawley and Stone, 1998; Bai and Stone, 2011) and Hall drift (Sano and Stone, 2002a b; Kunz and Lesur. 2013). The criterion also adequately describes the height of the boundary in stratified local simulations treating resistivity Turner and Sano, 2008, Okuzumi and Hirose, 2011).

Most assessments of the dead zone extent in protoplanetary disks included only Ohmic resistivity. However, ambipolar diffusion is more effective in the surface layers. The MRI turbulence's upper and outer edges tend to be set by ambipolar diffusion Perez-Becker and Chiang, 2011b, Bai, 2011a) or by the fields' stiffness at high magnetic pressure (Miller and Stone, 2000. Turner et al., 2010). Polycyclic aromatic hydrocarbon grains (PAHs) if found at abundances similar to those in Herbig Ae/Be disks would limit the turbulence to a thin layer ionized by the stellar FUV in the disk near the star (Perez-Becker and Chiang, 2011ab), but at the lower densities found further out, PAHs enhance magnetic coupling (Bai, 2011b).

Hall drift plays a critical role throughout much of the disk (Fig. 1), increasing or decreasing the estimated active column by orders of magnitude Wardle and Salmeron, 2012). However for $\Lambda_{H} \lesssim 5 v_{A} / c_{s}$ the Hall drift leads to most of the magnetic flux being confined into zonal regions in unstratified calculations, shutting down turbulent transport Kunz and Lesur, 2013). This has the potential to greatly reduce the extent of the MRI turbulent layer. The following section deals with the saturation of the MRI and the properties of the resulting flows. 

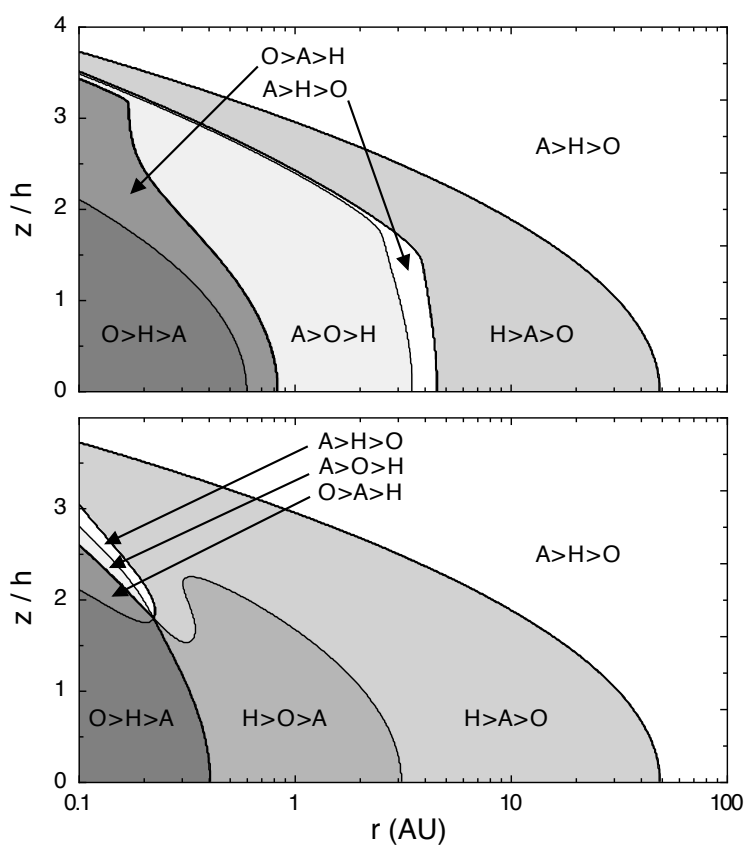

Fig. 1. - Effect of grains on magnetic diffusion and drift in the minimum-mass Solar nebula where $\Sigma=$ $1700(r / \mathrm{AU})^{-1.5} \mathrm{~g} \mathrm{~cm}^{-2}, T=280(r / \mathrm{AU})^{-0.5} \mathrm{~K}$ and $B$ is constant with height and chosen so $v_{A}=0.1 c_{s}$ at the midplane. The axes are the radius in AU and height in scale-heights. Shading indicates the ordering of the Ohmic, Hall and ambipolar diffusivities for a population of $0.1 \mu \mathrm{m}$ grains with $1 \%$ (top panel) or $0.01 \%$ (lower panel) of the gas mass, under ionization by $\mathrm{X}$-rays, cosmic rays and radioactivity.

\section{Saturation of MHD Turbulence}

While the last section covered the linear MRI, this one focuses on the non-linear evolution. By the mid1990s it was established that the MRI robustly leads to MHD turbulence transporting angular momentum outward (Hawley and Balbus, 1992, Hawley et al. 1995 . Brandenburg et al. 1 1995; Hawley et al. , 1996). However in recent years, our view of disk turbulence has been challenged by subtle effects both numerical and physical, thanks to faster computers and a better understanding of the microphysics. The purpose of this section is to review these findings and discuss the saturation amplitude of the turbulence. The amplitude is commonly measured by $\alpha$, the ratio of accretion stress to gas pressure. The stress $-B_{R} B_{\phi} / 4 \pi+\rho v_{R} \delta v_{\phi}$ depends on the density $\rho$ and the radial and azimuthal components of the magnetic field and velocity, the latter measured relative to the background orbital rotation (Hawley et al. 1995).

\subsection{Numerical Approaches}

Many of the codes now in use employ variations of the conservative Godunov scheme. This is the case for example in ATHENA Stone et al.
2008), Pluto (Mignone et al., 2007) and NirvanaIII (Ziegler, 2005, 2008). Alternatives include the pseudo-spectral code SNOOPY (http://ipag.osug. fr/\$ $\backslash$ sim\$glesur/snoopy.html) and the 6th-order finite difference PENCIL CODE (http://pencil-code. nordita.org), while ZEus (Stone and Norman, 1992, Hawley and Stone, 1995), which dominated the early days, is still used by some teams. With this wealth of methods, a large variety of setups has been developed for studying the MRI's non-linear evolution. Here we have space for only a sampling.

The most popular of all the approaches is the local shearing box model. The differential rotation is linearized in a small Cartesian volume rotating around the central object. Compressible and incompressible formulations are used and the vertical stratification can be included (Fig. 2). The shearing box is useful as a simplified framework for the dynamics. The problem is physically well-posed and boundary conditions are straightforward. High numerical resolutions can be reached and the consequences of dissipative processes investigated. We detail recent findings in $\$ \$ 3.2$ 3.5. Of course, the shearing box's advantages come at a cost: curvature terms are ignored and large-scale gradients are neglected. These limitations have motivated the development of global simulations treating the radial structure. Spatial resolution is sacrificed, and boundary conditions require care, but in other respects the realism is better and the results can be compared directly with observations. We review recent global findings in 3.6 .

A key aspect of all simulations is the magnetic field topology. In the shearing box this is particularly important, because the magnetic flux threading the domain is conserved over time due to the boundary conditions: for example, any net (domain-averaged) vertical field present initially remains for all time. Different configurations have been investigated: vertical and/or toroidal fields yield "net flux simulations" in which the ever-present magnetic flux is a permanent seed for turbulence. Configurations with vanishing mean magnetic field have also been investigated. In this peculiar situation, the flow must constantly regenerate its own magnetic field through a dynamo process.

\subsection{Ideal MHD Simulations}

Here we describe the lessons learned in recent years using local numerical calculations neglecting all explicit non-ideal magnetic effects. To further simplify the problem, the equation of state is most often taken to be isothermal. The flow then depends a priori on the magnetic field topology, box size, grid resolution, and whether the vertical stratification is included.

The special zero-net-flux or dynamo case has proven to be peculiar. In the absence of vertical stratification, MRI-driven turbulent activity decreases as resolution is increased (Fromang and Papaloizou, 2007, 


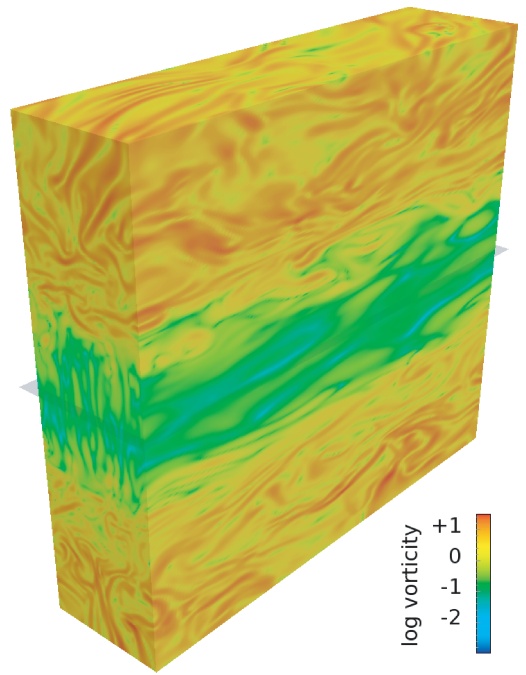

Fig. 2.- Stratified shearing-box calculation of a small patch of protostellar disk. The Ohmic resistivity is low enough for magneto-rotational turbulence only in the X-ray ionized layers on the disk's top and bottom surfaces. Colors indicate the vorticity $|\nabla \times \mathbf{v}|$ of the velocity $\mathbf{v}$ relative to the Keplerian shear. The ionized, turbulent layers show folded eddy structures, while the flow near the midplane is dominated by shearing waves Gressel et al. 2011.

Guan et al. 2009: Simon et al. 2009; Bodo et al. 2011, with no sign of converging to a well-defined value even at resolutions up to 256 cells per density scale height. This is a surprise because when extrapolated to infinite resolution, it suggests the flow remains laminar in the absence of a net magnetic flux. It is still not clear whether this result is a numerical artifact, as claimed early on (Fromang and Papaloizou, 2007) or is due to limitations of the shearing box (Kitchatinov and Rüdiger, 2010, Bodo et al., 2011)

Non-convergence, however, appears to be specific to the homogeneous shearing box without net magnetic field. When the density stratification is treated, numerical convergence is reached (Davis et al., 2010, Shi et al., 2010). It is unclear why this differs from the homogeneous case. A suggestion is that the episodic escape of toroidal magnetic fields revealed in the socalled butterfly diagram (Miller and Stone, 2000, Shi et al., 2010, Hirose and Turner, 2011) mediates a mean-field dynamo (Gressel, 2010). Care is needed because the grid resolutions in stratified simulations are limited, owing to the high Alfvén speeds in the disk corona which necessitate timesteps shorter than in the homogeneous case. The lack of convergence of turbulent transport also disappears when a net magnetic flux, toroidal (Guan et al. 2009) or vertical (Simon et al., 2009), threads the computational domain. Again the reason for the difference compared with zero- net-flux boxes has not been identified, even if it seems natural to relate it to the linear MRI modes in that case. With a net vertical magnetic field, the turbulent activity depends on the box's radial extent Bodo et al. 2008) and this comes from the linear MRI modes and how they are destabilized by parasitic instabilities.

Clarifying these issues will be tremendously valuable. However, regardless of its origins, the convergence issue highlighted in this section teaches us that the grid resolution in current numerical simulations affects the outcome in some cases, and thus dissipation should be treated explicitly. This is the topic of the following sections.

\subsection{MRI with Ohmic Resistivity}

To address the convergence issue, several investigations have included a constant Ohmic resistivity, $\eta$, often along with a constant kinematic viscosity $\nu$. The result that has emerged is that both dissipation coefficients are important: $\alpha$ is an increasing function of their ratio, the magnetic Prandtl number $P m=\nu / \eta$ (Fig. 3). This result is robust, having appeared in homogeneous shearing boxes with net vertical $($ Lesur and Longaretti, 2007, Longaretti and Lesur, 2010) and toroidal (Simon and Hawley, 2009) magnetic fluxes, with no net flux (Fromang et al., 2007) and in stratified boxes without net flux, though it appears weaker in that case (Simon et al. 2011a). As an example, in homogeneous boxes with a vertical magnetic field having $\beta=10^{3}, \alpha$ ranges from $2 \times 10^{-2}$ to $10^{-1}$ when $P m$ varies from 0.0625 to 1 , all else being equal. Recall that in the ideal MHD limit $(\sqrt{3.2}$ this case shows numerical convergence. Clearly, numerical and physical convergence are two different things and small-scale dissipation matters even in situations that appear numerically converged, at least over the limited range of Reynolds numbers that is numerically accessible.

These results raise questions about the flow structure in protoplanetary disks' inner parts where the resistivity is much larger than the viscosity (Balbus and Henri, 2008) $(P m \ll 1)$. The problem is this regime is numerically challenging to investigate as it requires extremely large spatial resolution: the smallest published value of $P m$ amounts to 1/16. Does $\alpha$ approach an asymptote at lower $P m$ ? If an asymptote exists, how small is $\alpha$ there, especially when stratification is included? These questions' broad implications make them a focus of ongoing research.

While these results highlight the properties of the MRI in protoplanetary disks' inner parts, they are not directly applicable to the planet-forming region of protoplanetary disks, where magnetic coupling is good only in the surface layers. In the scenario suggested early on by Gammie (1996), the disk is divided into a laminar equatorial "dead zone" and MRI-turbulent surface "active layers". Modeling this scenario, Fleming and Stone (2003) used an Ohmic diffusivity that is 


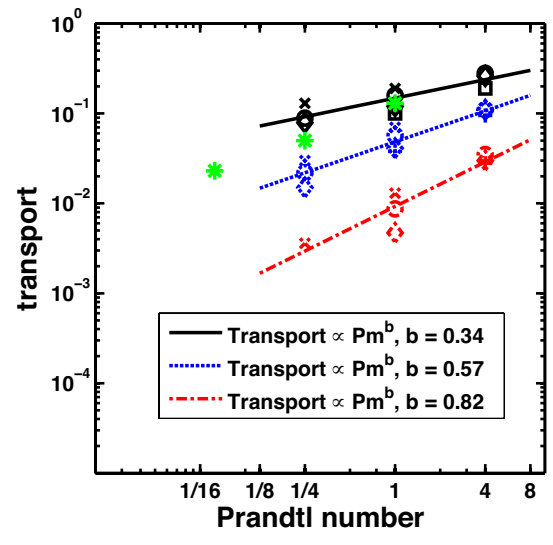

Fig. 3.- Turbulent angular momentum transport coefficient $\alpha$ vs. Prandtl number $P m$ and plasma $\beta$ for various Reynolds numbers. Solid, dotted and dotdashed indicate $\beta=10^{2}, 10^{3}$ and $10^{4}$. Squares, pluses, diamonds, circles and exes mark $R e=400,800,1600$, 3200 and 6400. A power-law fit is shown for each value of $\beta$. Stars mark 3 higher-resolution runs at $R e=20000$ and $\beta=10^{3}$ (Longaretti and Lesur, 2010).

a fixed function of time and height above the equatorial plane. The MRI is stabilized in the dead zone while the active layers are turbulent. The dead zone shows hydrodynamical stresses excited by sound waves propagating from the active layers, confirmed by Oishi and Mac Low (2009) among others. Another modification to the original scenario is that large-scale mean magnetic fields can diffuse downward to transport angular momentum within the dead zone itself Turner and Sano, 2008). The $\alpha$ values reported are around $10^{-4}$. Semi-analytic recipes are now available describing the vertical structure of such layered disks (Okuzumi and Hirose, 2011).

Two further aspects of the layered disk picture have been investigated intensively since the previous volume in this series. The first involves the changes over time in the Ohmic diffusivity profile as a function the local flow properties (Ilgner and Nelson, 2006b; Turner et al., 2007; Ilgner and Nelson, 2008). The second involves better treating the disk thermodynamics (Flaig et al. 2010, Hirose and Turner, 2011). The vertical distribution of heating differs significantly from that assumed in $\alpha$-models.

\subsection{MRI With Hall Effect}

The first investigation of the MRI with Hall effect was performed by Sano and Stone (2002a b) who found that MRI turbulence with both Hall and Ohmic terms was rather similar to MRI turbulence with the Ohmic term alone. The stress depended weakly on the Hall parameter, $\alpha$ increasing with $\eta_{H}$. However, Wardle and Salmeron (2012) pointed out that there was room for stronger effects in the Hall-dominated regime where the Hall term is the largest in the induction equation.

The problem was revisited by Kunz and Lesur (2013) who showed that under a Hall term 10-100× stronger than in Sano and Stone 2002b, the MRI develops into self-sustaining large-scale zonal magnetic field structures which produce only very weak angular momentum transport $\left(\alpha \sim 10^{-5}\right)$. The transition to this regime happens where $\Lambda_{H} \lesssim 5 v_{A} / c_{s}$. Therefore, even if the linear analysis shows fast growth at large scales, the instability does not necessarily lead to MHD turbulence and enhanced angular momentum transport. Note that these results are from unstratified shearing boxes. It is thus too soon to rule out Hall-dominated MRI as a source of turbulent transport in disks, since stratification is likely to modify these results.

\subsection{MRI With Ambipolar Diffusion}

Numerical implementation of ambipolar diffusion comes in two flavors. In the two-fluid approach, ions and neutrals are treated as separate fluids coupled by collisional drag, but typically not by chemistry, with the magnetic field carried by the ions. In the singlefluid approach the neutrals are the fluid, with the ions, whose density is determined by equilibrium chemistry, providing dissipation through ion-neutral drag. Generally speaking, the two-fluid approach applies to wellionized systems with slow recombination, while the single-fluid approach applies in the opposite ("strongcoupling") limit. Chemical modeling indicates the strong-coupling limit is almost always valid in protostellar disks (Bai, 2011a). Early on Mac Low et al. (1995) incorporated ambipolar diffusion in their singlefluid simulations, but a systematic study was lacking. Hawley and Stone (1998) studied the MRI with ambipolar diffusion using the two-fluid approach, so the results do not apply directly to protostellar disks.

Bai and Stone (2011) made a series of unstratified shearing-box simulations of the MRI with ambipolar diffusion in the strong-coupling limit. They systematically explored the ambipolar diffusivity, magnetic field strength and field geometry, finding consistent with linear analysis ( 2.3 ) that the MRI operates at any value of $A m$ as long as the field is weak enough that the most unstable mode fits within the disk scale height. The saturation level of the turbulence depends on field strength and configuration, and when ambipolar diffusion is strong $(A m<10)$, fields with comparable net vertical and toroidal components are best, while a pure toroidal field suppresses the MRI. Summarizing their results, the saturation level of the MRI turbulence with the most favorable field geometry is

$$
\alpha_{\max } \approx \frac{1}{2}\left[\left(\frac{50}{A m^{1.2}}\right)^{2}+\left(\frac{8}{A m^{0.3}}+1\right)^{2}\right]^{-1 / 2} .
$$

The next step has been to treat ambipolar diffusion 
in stratified simulations. Ambipolar diffusion is the dominant non-ideal MHD effect in low-density regions, including the surface layer of the inner disk together with the entire outer disk (\$2). For the inner disk at 1-10 AU, Bai and Stone (2013b) and Bai (2013) performed stratified shearing-box simulations that include both Ohmic resistivity (dominant in the midplane) and ambipolar diffusion (dominant at the disk surface) with diffusion coefficients based on the equilibrium chemistry calculations of Bai (2011a), as well as the FUV ionization model of Perez-Becker and Chiang (2011a). They found that without net vertical magnetic flux, the system evolves into extremely weak turbulence in the FUV layer, far too weak to drive appreciable accretion. When a net vertical magnetic flux is included, even if the initial field configuration is subject to the MRI, the instability is suppressed and the disk's whole vertical extent relaxes into a laminar state, while a wind is launched and efficiently extracts angular momentum from the disk (\$4). In both cases the MRI is suppressed by ambipolar diffusion. For the outer disk at $\gtrsim 30$ AU, Simon et al. $(2013 \mathrm{a}$ b) performed similar calculations and found first that net vertical magnetic flux is essential otherwise the resulting MRI turbulence is too weak. In the presence of net vertical flux, the turbulence is stronger and takes place mainly in the surface FUV layer. Both results qualitatively agree with expectations from unstratified simulations $(B a i$ and Stone, 2011), except that with increasing net vertical magnetic flux, large-scale field comes to dominate over turbulent field as the driver of angular momentum transport.

\subsection{Global Calculations}

Local simulations are invaluable for studying turbulence, but their reach is limited. Thus several teams have embraced the challenges of global simulations. Resolutions do not yet approach those achieved in the shearing box, where $>100$ cells per scale height is commonplace. Consequently it is difficult at present to include small-scale dissipation in global work. The only successful example is Dzyurkevich et al. (2010), where the Ohmic diffusion is treated. Given all the subtleties uncovered when the non-ideal effects were taken into account in shearing boxes, doubts remain about our ability to characterize the saturation of the turbulence through global calculations. The global results to date should therefore be interpreted with care. Nevertheless, progress has been made thanks to growing computer power and there have been claims that the bestresolved models are numerically converged (Sorathia et al. 2012). Of course the lesson of the shearing box is that numerically-converged simulations do not necessarily yield the correct stresses, as these can depend on effects not treated (Hawley et al. 2013 ).

The basic result of global simulations is boring and reassuring at the same time: the transport is consistent with shearing box simulations under similar conditions (Fromang and Nelson, 2006). The turbulent velocity dispersion increases with height Fromang and Nelson 2009, Flock et al., 2011) as in shearing boxes (Miller and Stone, 2000). There is growing evidence that largescale flow features are similar in wide shearing boxes (Simon et al. , 2012) and global calculations (Beckwith et al., 2011; Flock et al., 2012). Likewise, the zonal flows, axisymmetric variations in the orbital speed that persist for many orbits, seem to be a natural outcome of MHD turbulence in both setups Johansen et al. 2009, Uribe et al., 2011, Dittrich et al. 2013).

Some issues can only be addressed using global calculations. For example Sorathia et al. (2010) reported that small patches of disk behave as if threaded by a vertical net field, even if the total vertical flux threading the disk as a whole vanishes. Such fields connect the disk atmosphere with its equatorial plane, suggesting that coronal magnetic fields are central in determining the accretion flow (Beckwith et al. 2011). Protoplanetary disks' large scale flow, important for our understanding of particle transport, remains poorly known. The prediction from traditional $\alpha$-models of a meridional circulation, with matter flowing outward near the midplane while accretion proceeds through the upper layers, fails in fully turbulent (and thus unrealistic) disks (Fromang et al. 2011, Flock et al. 2011). It is likely that mixing within disks made up of both active and dead zones differs from the models published so far.

Besides the resolution issue already mentioned, global simulations suffer from two limitations of special importance. First, the equation of state is typically locally isothermal with no explicit heating and cooling. These are potentially important for the flow in the disk corona, the structure of the disk interior, and the dead zone nearest the star. Second, no published global simulation of turbulent protoplanetary disks yet includes a net vertical magnetic flux. Given the differences between shearing-box results with and without vertical fluxes ( $\$ 3.5$ and 4), such simulations would be extremely valuable and should be a focus in coming years.

\subsection{Outstanding Issues}

While small-scale dissipation has been (and still is!) studied in detail, the results have mainly taught us to be humble: more than 20 years after the discovery of the MRI, we still do not know how the turbulence saturates as a function of parameters such as the surface density, temperature and magnetic flux.

The question of how disk dynamos work is still debated. The results of the last few years suggest that brute force is of limited interest due to the enormous resolutions required. An interesting alternative is provided by the discovery of limit cycles near marginal stability (Herault et al. 2011), which might bear some 
significance for fully developed turbulence.

Perhaps most importantly for the transport, all of the results based on the MRI linear properties agree in their predictions that protoplanetary disks are MRIinefficient at distances from the star between about 1 and 10 AU (Turner and Drake, 2009, Bai, 2011a b. Perez-Becker and Chiang, 2011a b; Mohanty et al. 2013: Dzyurkevich et al. , 2013). In this section, we have seen that many predictions of the linear stability analyses carry over to the non-linear regime, with the notable exception of the Hall effect. Together these results suggest that something is missing in our understanding of the basic processes driving the accretion flows in the planet-forming region. This has revived interest in an old alternative transport mechanism, namely disk winds, the focus of the next section.

\section{Disk-Driven Winds and MHD Turbulence}

In previous sections we explored the possibility of driving accretion by transporting angular momentum inside the disk through the MRI. However, this is not the only way accretion can occur. An alternative is to extract the angular momentum vertically by applying a torque at the disk surface. Such a torque usually comes from a large-scale wind driven by magnetic processes. This section is dedicated to these winds' launching and their impact on accretion in protoplanetary disks. Jets formed as disk winds propagate to large distances are not discussed in this section. The interested reader may refer to the chapter by Cabrit et al.

To understand how a cold wind can drive accretion, consider angular momentum conservation in a thin disk

$$
\begin{aligned}
\overline{\rho v_{R}} \frac{\partial \Omega R^{2}}{\partial R}= & \frac{1}{R} \frac{\partial}{\partial R} R^{2}\left[\overline{B_{\phi} B_{R}}-\overline{\rho v_{\phi} v_{R}}\right] \\
& +R\left[B_{\phi} B_{z}-\rho v_{\phi} v_{z}\right]_{z=-h}^{+h},
\end{aligned}
$$

where $\Omega$ is the Keplerian frequency, $h$ is the disk scaleheight and overbars mean $\int_{-h}^{h} \mathrm{~d} z$. Eq. 3 shows that accretion (negative $\overline{\rho v_{R}}$ ) can be driven either by angular momentum transport inside the disk (first term on the right-hand side) or by a torque exerted at the disk surface (second term on the right-hand side). It is the second term which connects winds to accretion in the bulk of the disk. Eq. 3 also shows that a magnetically driven wind can easily dominate accretion in thin disks. Neglecting the kinetic term, one easily deduces that the turbulent torque $\sim h B_{\phi} B_{R}$ while the wind torque $\sim R B_{\phi} B_{z}$. Assuming $B_{\phi} B_{R} \sim B_{\phi} B_{z}$ within the disk, one finds that the torque due to the wind is $\sim R / h \times$ larger than the torque due to turbulence.

\subsection{Launching Mechanism}

Protoplanetary disks are thin $(h / R \lesssim 0.1)$ and dynamically cold, so winds cannot be launched exclusively by thermal processes at the disk surface. Driving a wind involves accelerating the flow to escape speed, which requires some extra power source. The power can come either from heating the gas as it is ejected (see the chapter on photoevaporation in this book) or from rotational energy extracted using a large-scale magnetic field, a process known as magnetocentrifugal acceleration (Blandford and Payne, 1982). The latter is extensively described in the literature (see Spruit 1996 for an introduction) so we here give only a brief qualitative picture.

Consider a frame co-rotating with the disk at radius $R_{0}$ from the central star. In this frame, fluid parcels feel an effective potential combining the gravitational and centrifugal potentials. If poloidal $(R, z)$ magnetic field lines act as rigid wires for fluid parcels, then a parcel initially at rest at $\left(R=R_{0}, z=0\right)$ can undergo a runaway if the field line to which it is attached is more inclined than a critical angle. Along such a field line, the effective potential decreases with distance, leading to an acceleration of magnetocentrifugal origin. This yields the inclination angle criterion $\theta>30^{\circ}$ for the disk-surface poloidal field with respect to the vertical. Here fluid parcels rotate at constant angular velocity and so increase their specific angular momentum.

Magnetic field lines act as rigid wires so long as the poloidal flow is slower than the local Alfvén speed that is, most of the energy is contained in the magnetic fields. The accelerating flow eventually reaches a poloidal speed exceeding the Alfvén speed, at a location known as the Alfvén point. Above here, magnetocentrifugal acceleration ceases and the flow winds up the field into toroidal coils. From this point on, fluid parcels rotate at constant specific angular momentum.

Angular momentum is thus extracted from the disk first by the magnetic torque at the disk surface $\left(B_{\phi} B_{z}\right.$ in equation 3 ) and stored in the magnetic field's toroidal component. As acceleration proceeds, the angular momentum is slowly transferred to the ejected material until the Alfvén point is reached. Winds therefore efficiently drive accretion by purely and simply removing the angular momentum from the disk. Moreover, one can see easily that the efficiency of this process is directly connected to the disk's magnetic field strength, a stronger field leading to a bigger torque and therefore faster accretion.

We have seen that a wind implies an accretion flow inside the disk. The poloidal field lines threading the flow are accreted too, if ideal MHD is valid. Such a flow must soon stop once a large magnetic flux accumulates near the disk's center. A long-lived wind requires the flow to slip past the field lines. This is usually modeled assuming the disk is either turbulent, producing a turbulent diffusivity (Ferreira and Pelletier, 1993. 1995: Ferreira, 1997), or subject to strong ambipolar diffusion (Wardle and Königl, 1993, Königl et al. 2010, Salmeron et al., 2011), a reasonable assumption at large distances ( $\gtrsim 10 \mathrm{AU})$. 
These very simple arguments show three basic requirements for a steady magnetocentrifugal wind:

- The field is strong enough for the poloidal component to act as a rigid wire. This means that at the base of the wind the plasma $\beta \lesssim 1$, where the magnetic pressure is that in the mean poloidal field. Fluctuations due to turbulence can exceed the mean field, but are not directly relevant for the existence of a steady wind.

- The field is sufficiently inclined, with $\theta>30^{\circ}$.

- Accreting gas slips through magnetic field lines, via either turbulent diffusion, or Ohmic or ambipolar diffusion.

\subsection{Local Disk Wind Solutions}

A natural question is whether the wind can coexist with turbulence inside the disk. However, numerically spanning both the small turbulent eddy scales and the large global scales associated with the wind propagation is a challenge. Two complementary approaches are used: (1) Local methods such as the shearing box involve treating the disk up to a few scale heights while neglecting the global geometry. (2) The global approach means approximating turbulence by some crude mean-field method. We focus here on the first approach since our topic is disk transport, but we also discuss the connection to global solutions to demonstrate the limitations of local solutions.

The shearing box outlined in 83 is shear-periodic in the radial or $x$-direction and periodic in the toroidal or $y$-direction. The vertical or $z$-boundaries have outflow conditions to cope with winds. This approach has several limitations:

Symmetries: The shearing box does not specify whether the central object lies at $x \rightarrow+\infty$ or $x \rightarrow-\infty$, leaving the two equivalent. This symmetry is apparent in shearing box results, which can exhibit winds bent toward either large or small $x$.

Magnetic flux transport: Because curvature terms are neglected in the shearing box, magnetic flux tubes can cross the box again and again without the fields piling up anywhere, since the flux escaping matches that entering on the box's far side. In other words, shearing boxes allow spurious solutions with constant $E_{\phi} \neq 0$. This unrealistic situation should be kept in mind when one compares local and global wind solutions.

Boundary conditions: The third and most stringent limitation comes from the effective potential (gravitational plus centrifugal) in the shearing box being a local expansion of the real poten- tial around $R_{0}$ of the form

$$
\psi \propto-\frac{3}{2} x^{2}+\frac{1}{2} z^{2},
$$

where $x$ and $z$ are respectively local equivalents of $R-R_{0}$ and $z$. Clearly this potential does not allow a particle to escape to $z \rightarrow \infty$ if $x$ is kept finite. Because magnetocentrifugal wind solutions cannot propagate radially past the Alfvén radius, disk winds cannot be gravitationally unbound in a finite shearing box. The winds that nevertheless appear owe their existence to the outflow vertical boundary conditions.

Below we show how these limitations affect the numerical results.

All the local numerical wind solutions obtained in recent years can be understood in the framework of the magnetocentrifugal acceleration mechanism described above. Here we distinguish "strong field" solutions, for which magnetic and thermal pressure are near equipartition $\beta_{\text {mid }} \sim 1$ in the midplane, from "weak field" solutions where thermal pressure dominates at the midplane $\beta_{\text {mid }} \gg 1$ (but not necessarily in the disk corona). We furthermore separate the solutions by the non-ideal effects included.

Ideal and weakly resistive winds Ideal MHD solutions are the simplest obtainable, but of course neglect the non-ideal effects which can be important in protoplanetary disks. Moreover, under ideal MHD the numerical convergence of MRI turbulence can be an issue (\$3). To address these limitations, some solutions have been computed in the resistive MHD framework. A weak resistivity and viscosity seem not to affect the wind dynamics (Fromang et al., 2013).

The first ideal-MHD solutions exhibiting both a disk wind and the MRI on weak fields $\left(\beta_{\text {mid }} \gg 1\right)$ were obtained by Suzuki and Inutsuka (2009). These involve weak poloidal fields $\beta_{\text {mid }}=10^{6}-10^{4}$. Standard MRI turbulence appears and a short-lived magnetocentrifugal wind slowly empties the domain since the shearing box boundaries allow no replenishment by accretion flow. Because the midplane fields are weak, the wind is launched about two scale-heights up, so that $\beta \sim 1$ at the launching surface as expected from the phenomenology presented above. The wind mass loss rate $\dot{M}_{W}$ increases steeply as $\beta$ decreases, almost emptying the box after 75 rotations for $\beta=10^{4}$. Suzuki et al. (2010) explored the consequences of such a wind for global disk models, demonstrating that a wind could empty the inner disk and create an inner hole. Bai and Stone (2013a) explored stronger fields up to $\beta=100$. They confirmed some of the Suzuki and Inutsuka (2009) results, showing that $\dot{M}_{W} \propto 1 / \beta$ holds for the $\beta$ values they explored. However, Fromang et al. (2013) and Bai and Stone (2013a) demonstrated that $M_{W}$ was highly sensitive to the domain 
height: $\dot{M}_{W} \propto 1 / L_{z}$ where $L_{z}$ is the box vertical size. This is a clear signature of the third shearing box limitation detailed above. Box simulations yield unreliable results for the mass flow rate extracted by disk winds and the efficiency of disk dispersal.

The shearing box's extra symmetries also allow the wind to switch erratically between ejection toward larger and smaller radii (Fromang et al., 2013, Bai and Stone, 2013b). The configuration switchings happen independently above and below the disk. For example, the upper disk can drive a wind toward larger radii while the lower disk drives a wind toward smaller radii. Such artificial outcomes are unsatisfactory for a global overview of the wind structure.

The strong-field regime $\beta_{\text {mid }} \sim 1$ is well-studied for jet formation because it is the only one where selfsimilar global wind solutions exist (Ferreira, 1997). This regime has also been explored in the local approximation. Ogilvie (2012) obtained 1-D quasi-steady strong-field wind solutions for which $\dot{M}_{W}$ decreases sharply when $\beta$ decreases. Wind solutions possibly even cease to exist for sufficiently strong fields $\left(\beta_{\text {mid }} \simeq 0.3\right)$. This study was revisited in 3 -D by Lesur et al. (2013) in a regime where wind solutions of Ogilvie (2012) coexist with large-scale MRI modes (Latter et al. 2010). They showed that MRI modes naturally evolve into quasi-steady wind solutions when the field is strong enough $(\beta \sim 10)$, making a natural connection between the MRI and strong winds. However, similarly to the weak field case, $\dot{M}_{W}$ depends on the box vertical extent, casting doubt on the conclusions. Moll (2012) and Lesur et al. (2013) showed that the quasi-steady wind solutions of Ogilvie (2012) were radially unstable, producing time-dependent wind solutions. Several mechanisms potentially lead to unstable winds (Lubow et al. 1994b, Lubow and Spruit, 1995: Cao and Spruit, 2002). However none seems entirely compatible with numerical results Moll, 2012, Lesur et al., 2013).

In all these numerical solutions, the toroidal electromotive force $E_{\phi} \neq 0$ and magnetic flux tubes accrete. This is to be expected since the solutions are computed in ideal or weakly resistive regimes.

Winds with ambipolar diffusion and Hall effect The Hall effect and ambipolar diffusion are believed to dominate in much of protoplanetary disks (\$2). Ambipolar diffusion is specially important since it lets the gas cross magnetic field lines rather than sweeping them along. In principle this avoids the flux accumulation problem faced by the ideal-MHD and weakly resistive models described in $\$ 4.2$.

Local solutions including both Hall effect and ambipolar diffusion were initially presented by Wardle and Königl (1993) who treated the ions, electrons and neutrals as independent fluids coupled together by a drag force. With this approach, the neutrals (which make up most of the mass) can accrete without advecting field lines (which are attached to the charged species). Wardle and Königl (1993) demonstrated steady wind solutions in the $\beta_{\text {mid }} \simeq 1$ regime, both with and without accreting magnetic field lines. Surprisingly, solutions with and without magnetic accretion are similar, indicating the magnetically accreting solution presented in 4.2 might be qualitatively correct.

Comparable winds can be launched when the Hall and Ohmic diffusivities are large Königl et al. 2010. Salmeron et al. 2011). Interestingly, the mass-loss rate and torque depend on the poloidal field polarity (i.e. the sign of $\mathbf{B} \cdot \boldsymbol{\Omega}$ ). In particular, some wind solutions are obtained only for positive polarities.

The problem of outflows in the presence of ambipolar diffusion was revisited by Bai and Stone (2013b). Using a complex chemical network to compute ambipolar and Ohmic diffusivities, they found midplane ambipolar diffusion several orders of magnitude stronger than anticipated by Wardle and Königl (1993) and concluded that non-ideal effects suppress MRI turbulence at 1 AU. However, even poloidal fields as weak as $\beta_{\text {mid }}=10^{5}$ trigger a quasi-steady wind starting about 4 scale-heights above the midplane, where ambipolar diffusion is significantly reduced thanks to FUV ionization. While the shearing box is ambiguous regarding the direction to the star, global magnetocentrifugal wind solutions generally have the horizontal components of the magnetic field changing sign across the disk. In the local weak-field ambipolar-dominated calculations, the horizontal field flips in a thin layer offset from the midplane by several scale-heights. This layer, a fraction of a scale-height thick, receives the wind torque and carries the entire accretion flow. Based on these results, Bai (2013) proposed that the accretion rates in the planet-forming region at $\sim 1-10$ AU can be explained by such a steady wind alone, the bulk of the disk being essentially quiet. The ambipolar-diffusiondominated outer disk is likely MRI turbulent but can also launch an outflow (Simon et al. 2013b).

Connecting local and global solutions In one of the first attempts at connecting local and global solutions, Wardle and Königl (1993) matched their local results to a global Blandford and Payne (1982) wind, ruling out some of the local solutions. Similarly, Lesur et al. (2013) compared their local solution against global solutions computed à la Ferreira and Pelletier (1995). There was qualitative agreement but significant differences remained, e.g. in mass loss rate and $\beta_{\text {mid }}$. Interestingly they found no global solutions for fields as weak as they considered in the local solutions, suggesting that if a global wind exists for given parameters, then the local approach will catch the right solution. However, the local approach might also catch 
solutions which simply do not exist in global geometry. These spurious winds are impossible to distinguish from "real" winds solely by a local approach. Future work on this topic will therefore have to extend the recent findings on local solutions to global geometries.

\subsection{Global Disk Wind Solutions}

To overcome the limitations of local solutions, one can perform global calculations. Here however a scale separation problem occurs. On one hand, any turbulence must be resolved. On the other hand, the solution must be computed far from the disk in the vertical direction (typically to 10 s or even 100 s of disk radii). For this reason, all global disk wind solutions have been obtained in a 2.5-D approximation with turbulent motions either ignored or treated as viscosities and resistivities. This approximation is brought into question by $3-\mathrm{D}$ results on torus accretion by a black hole (Beckwith et al. 2009). The main difficulties with the global models are two-fold:

- They need a strong magnetic flux in the disk midplane $\left(\beta_{\text {mid }} \sim 1\right)$, which can either build up as flux accretes from the outer disk (a scenario with several problems, see Lubow et al. 1994a) or be put in place when the disk forms (in which case the entire question of jet formation is linked to the disk formation scenario).

- They rely on strong resistivities. A resistivity $\eta_{t}$, supposed to mimic the local effects of 3-D turbulence, is quantified by the parameter $\alpha_{m}$ in $\eta_{t}=\alpha_{m} c_{s} H$. To avoid magnetic flux accumulating, these models require $\alpha_{m} \sim 1$. However no physical mechanism justifies such a high turbulent diffusivity with $\beta_{\text {mid }} \sim 1$. In particular, MRI turbulence is believed to produce at best $\alpha_{m} \sim 0.1$ (Lesur and Longaretti, 2009).

The behaviour of global models as a function of the turbulent resistivity was investigated by Zanni et al. (2007) in 2.5-D simulations. For $\alpha_{m}=1$ and $\beta_{\text {mid }}=1$, they found quasi-steady ejection resembling the selfsimilar models of Ferreira (1997). Lower values of $\alpha_{m}$ invariably lead to unsteady ejection, probably due to episodes when magnetic flux accretes followed by sudden releases of the flux in numerical reconnection or ejection events. This indicates that ejection is possible for weak diffusivities, even though the resulting winds and jets are likely to be highly variable.

Issues of disk magnetisation were studied by Tzeferacos et al. (2009) and Murphy et al. (2010). They demonstrated the possibility of launching unsteady magnetically-driven winds up to $\beta_{\text {mid }} \sim 500$. However these models rely on large diffusivities with $\alpha_{m} \simeq 1$ which are probably too high to be realistic if turbulence is driven solely by the MRI. They also pointed out the sensitivity of the mass loading rate (fraction of the mass which is ejected in the wind) to the resolution. The high mass loading rate achieved in these simulations is likely due to numerical diffusion of gas at the top of the disk, where the launching occurs.

All the models considered above are "cold", with the turbulent heating canceled by radiative cooling. Tzeferacos et al. (2013) showed explicitly that if weak turbulent heating is not balanced by radiative cooling at the disk surface, the mass loading rate and ejection efficiency are significantly affected. This demonstrates the sensitivity of winds to thermodynamics near the launch point, which must be modeled accurately if we are to predict winds' large-scale properties and match jet observations (see chapter on jets by Frank et al. in this book).

\subsection{Outstanding Issues}

The new disk wind scenario differs significantly from the $\beta_{\text {mid }} \simeq 1$ picture investigated in the 1990s. The magnetization is weak, with $\beta_{\text {mid }} \sim 10^{4}$, and the ambipolar diffusivity varies with height. This scenario is still developing and global modeling may soon clarify the picture. Outflowing material could shield the disk from ionizing stellar photons (Ferro-Fontán and Gómez de Castro, 2003, Panoglou et al., 2012), preventing wind launching. The winds' sensitivity to radiative transfer and chemistry must be investigated further. Finally we note that this scenario is unlikely to explain the protostellar jets launched within $1 \mathrm{AU}$, where non-ideal MHD effects are weak thanks to thermal ionization.

\section{Self-Gravitating Turbulence}

Self-gravitating, non-axisymmetric structures transport angular momentum with a flux density

$$
W_{r \phi}=\int d z\left(\frac{g_{R} g_{\phi}}{4 \pi G}+\rho v_{R} \delta v_{\phi}\right)
$$

where $g_{R}$ and $g_{\phi}$ are the radial and azimuthal components of the gravitational acceleration. This transport is non-local in the sense that it can couple together parts of the disk that are widely separated in radius (Balbus and Papaloizou, 1999). Gravitational torques may, however, be described by an effective $\alpha$, if the characteristic scale $\lambda_{\text {char }}$ is small compared to the local radius (Lodato and Rice, 2004, 2005, Vorobyov and Basu, 2009, Cossins et al., 2009; Forgan et al., 2011, Michael et al. (2012) or equivalently the disk mass is small compared to the star (Vorobyov and Basu, 2010).

Self-gravitating disk structures are detectable in the millimeter continuum and lines in nearby $(d \lesssim 150 \mathrm{pc})$ systems with ALMA (e.g. Cossins et al., 2010, Douglas et al., 2013), although self-gravitating disks are likely to be found in young, short-lived systems, and are therefore expected to be rare. If they are found, torques and a disk evolution timescale can be measured directly from a surface density map, e.g. Gnedin 
et al. (1995), and molecular line observations may allow us to measure the ratio of vertical to in-plane velocity dispersion well enough to separate the values expected for gravitationally-driven and MRI-driven turbulence (Forgan et al. 2012).

When is the disk self-gravitating? Self-gravity becomes important when the Toomre parameter

$$
Q \equiv \frac{c_{s} \kappa}{\pi G \Sigma} \sim 1
$$

where $c_{s}$ is the sound speed, $\kappa$ the epicyclic frequency which $\simeq \Omega$ in a Keplerian disk, and $\Sigma$ the surface density. If we define the scale height $H \simeq c_{s} / \Omega$ and the disk mass $M_{d} \equiv \pi R^{2} \Sigma$, then we can rewrite $Q$ to obtain the useful relation $Q=(R / H) M_{d} / M_{*}$.

The $Q$ parameter describes the balance between the stabilizing influence of pressure $\left(c_{s}\right)$ and rotation $(\kappa)$ and the destablizing influence of self-gravity $(G \Sigma)$. Pressure and rotation are weakest in comparison to self-gravity at a characteristic wavelength

$$
\lambda_{c} \equiv \frac{2 c_{s}}{G \Sigma}=2 \pi Q H
$$

Measuring this characteristic scale in a face-on disk may then be a direct probe of the surface density, through $Q$; numerical experiments show that even in the non-linear regime much of the power is concentrated near $\lambda_{c}$ (Cossins et al., 2009).

The formal analysis leading to the conclusion that $Q>1$ implies stability has formal limitations: it relies on an axisymmetric analysis of a zero-thickness disk, and requires that $\lambda_{c} \ll r$ (it is a local, or lowest order $W K B$, analysis). But a self-gravitating disk is most responsive to non-axisymmetric perturbations; finite thickness is stabilizing Goldreich and LyndenBell, 1965, Mamatsashvili and Rice, 2010); and longwavelength modes can be stabilized or destabilized by global features in the disk (e.g. Meschiari and Laughlin, 2008). In the end, a good rule of thumb is that $Q \lesssim 2$ disks are strongly self-gravitating.

The outcome of gravitational instability depends on how the disk is driven unstable. In one commonly analyzed model the disk is driven unstable by cooling that is characterized entirely by the timescale $\tau_{\text {cool }}$ Gammie, 2001), or equivalently by the dimensionless $\beta_{c}=\tau_{\text {cool }} \Omega$. Then there is a $\beta_{\text {crit }}$ Shlosman and Begelman, 1987) dividing models that fragment immediately $\left(\beta_{c}<\beta_{\text {crit }} \simeq 3\right)$ from those that enter a quasi-steady, or gravito-turbulent, state at $\beta_{c}>\beta_{\text {crit }}$. Since cooling times typically decrease outward in protoplanetary disks, this implies that there is a critical radius at a few tens of AU beyond which a massive disk can fragment (Matzner and Levin, 2005, Rafikov, 2005). The disk can also be driven unstable by mass loading via infall. In this case whether the disk fragments depends on the ratio of infall to accretion timescales (e.g. Boley, 2009, Rafikov, 2009, Kratter et al., 2010, Harsono et al. 2011).
In a gravito-turbulent state the disk may settle into a condition of marginal stability, with $Q$ slightly greater than the marginally stable value Paczynski, 1978. Vorobyov and Basu, 2007). Transport in the gravito-turbulent state can be described by an effective $\alpha$, and is predominantly local, if $\lambda_{c} / R \lesssim 1$. If angular momentum transport can be described by an effective $\alpha$, its value is $(4 / 9) \beta_{c}^{-1} /(\gamma(\gamma-1))$ Gammie, 2001). Then a minimum $\beta_{c}$ implies a maximum $\alpha$ in the gravito-turbulent state; at larger values the disk fragments. The $\alpha\left(\tau_{\text {cool }}, \Omega\right)$ relationship also applies to more complicated cooling laws, but if there are orderunity variations in the surface density then it may be difficult to estimate $\tau_{\text {cool }}$.

Angular momentum transport will not cease if the disk fragments. Even low-mass bound objects will generate wakes in a self-gravitating disk due to tidal interactions (Julian and Toomre, 1966) and superposition of these wakes transports angular momentum Johnson et al. 2006). Massive, embedded objects can lead to rapid disk evolution (e.g. Krumholz et al., 2007, Vorobyov and Basu, 2010), or a complicated interplay between disk and planetary orbital evolution (Boley. 2009, Michael et al., 2011).

The critical value of $\beta_{c}$ remains unclear (Meru and Bate, 2011). Numerical outcomes are sensitive to numerical diffusion (Paardekooper et al. 2011. Paardekooper, 2012; Meru and Bate, 2012), the use of 2-D vs. 3-D models, gravitational softening (Müller et al., 2012), cooling with realistic opacities (e.g. Johnson and Gammie, 2003), and perhaps most important, irradiation (e.g. Matzner and Levin, 2005; Clarke et al. , 2007; Cai et al. 2008; Rice et al., 2011; Forgan and Rice, 2013), which at the least increases the temperature and therefore stabilizes the disk.

The most surprising new discovery about fragmentation is the finding by Paardekooper (2012) (see also Hopkins and Christiansen, 2013), stimulated by work of Meru and Bate (2011), that a 2-D local disk model can still fragment for $\beta_{c} \gg \beta_{\text {crit }}$, if one waits long enough! This is illustrated in Fig. 4. showing an apparently steady gravito-turbulent state later fragments at high enough resolution. The result is best described as stochastic fragmentation: for $\beta_{c}>\beta_{\text {crit }}$ the disk has a finite probability of producing a bound object per unit area per unit time. It is not yet known whether this extends to models in $3-\mathrm{D}$, or with more realistic cooling. Stochastic fragmentation may revive direct planet formation by gravitational instability for disks with $\beta_{c}>\beta_{\text {crit }}$ (e.g. Boss, 1997), or it may imply a $\beta_{\text {crit,eff }}>\beta_{\text {crit }}$ where the fragmentation time is the disk lifetime.

Evidently there are many open questions about angular momentum transport by gravitational instability. We think two in particular merit further exploration: (1) magnetized, self-gravitating disks. Does magnetically driven turbulence (in surface layers, or 


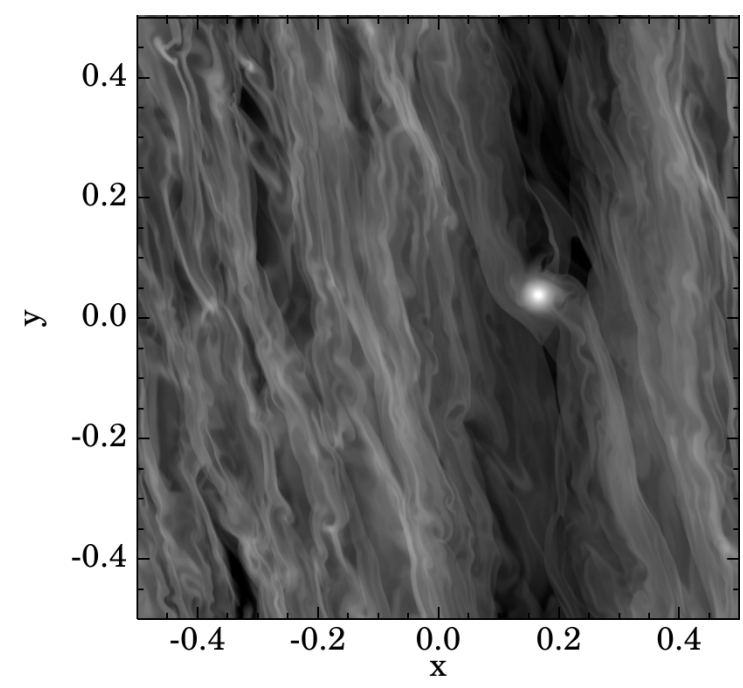

Fig. 4.- A 2-D (razor-thin) numerical model of a cooling, gravitationally unstable disk with $\beta_{c}=5$ is in an apparently steady gravito-turbulent state at $\Omega t=100$ but by $\Omega t=400$ produces a bound object as shown. Shading indicates surface density in units of the mean. From Paardekooper (2012).

throughout the disk) enhance or limit the development of bound structures in protoplanetary disks? Pioneering simulations (Fromang, 2005) need to be revisited at higher numerical resolution; (2) thermodynamically self-consistent disks with realistic opacities and external irradiation. While much work has already been done (Boss, 2007b, Cai et al., 2008, Steiman-Cameron et al. 2013) tracking cooling inside proto-fragments requires very high spatial resolution.

\section{Hydrodynamical Processes or Disk Weather}

Our understanding of the flows triggered solely by gas pressure and viscous forces has advanced greatly since the previous book in this series. Giant vortices, which may accelerate the planet formation process (Barge and Sommeria, 1995, Tanga et al. 1996 , Klahr and Bodenheimer, 2006), have gained observational support through ALMA observations of emission perhaps from mm-sized particles concentrated to one side of a young star (van der Marel et al., 2013). The feature resembles predictions of the vortices' appearance by Wolf and Klahr (2002) but has higher contrast since the big particles concentrate more strongly than the sub- $\mu \mathrm{m}$ grains in the model (Lyra and Lin, 2013).

Hydrodynamical turbulence is less robust than the MRI and self-gravity - otherwise it would have appeared in numerical models years ago. When magnetic fields couple to the gas, the MRI governs the flow Lyra and Klahr, 2011, Nelson et al., 2013). Thus the most promising sites for non-magnetic instabilities are the magnetically-decoupled dead zones. It seems likely that the Solar nebula at 1-10 AU from the young Sun was a suitable location.

Since protostellar disks have enormous Reynolds numbers $R e>10^{9}$, it might seem that their orbital shear would easily lapse into turbulence. However as rotating fluids, the disks are a special case of the Taylor-Couette flow, which is linearly stable when the angular momentum increases with radius, as it does on the Keplerian rotation curve. This is embodied in the Rayleigh stability criterion $\partial_{R}\left(\Omega^{2} R^{4}\right)>0 \sqrt{\text { Rayleigh }}$ 1917 ) at radius $R$ and rotation frequency $\Omega$. Based on contradictory Taylor-Couette experiments spanning almost a century (Taylor, 1923, Wendt, 1959, Taylor, 1936, Schultz-Grunow, 1959, Lathrop et al., 1992 , Richard and Zahn, 1999, Ji et al. 2006, Paoletti et al. 2012 Schartman et al., 2012), disks could be nonlinearly either stable or unstable. Yet the experiments depart significantly from astrophysical disks, especially at the vertical boundaries. The containers' upper and lower lids are argued to drive any turbulence observed in the experiments Avila, 2012). Also, even if accretion disks become turbulent at some critical Reynolds number above the maximum $R e \sim 2 \times 10^{4}$ achieved in numerical simulations, the resulting turbulent viscosity must still be relatively low, in fact inversely proportional to the critical Reynolds number Lesur and Longaretti, 2005). Thus we are compelled to investigate instabilities that occur under flow conditions relevant to protostellar disks and can be studied numerically.

Thermal convection perpendicular to the disk's midplane was once a favorite to cause turbulence. Yet it suffers a self-consistency problem, producing too little heating to sustain the vertical entropy gradient that drives it (as reviewed by Klahr, 2007). Convection pumped artificially by heating the midplane can however transport angular momentum outward (Klahr and Bodenheimer, 2003, Lesur and Ogilvie, 2010).

More promising are three classes of instabilities driven by radial stratification. We consider in turn the gradients in pressure, temperature and entropy. (1) Radial pressure gradients shift the rotation profile away from Keplerian. (2) Radial temperature gradients with Coriolis forces yield vertical shear, or orbital speed gradients in height, called "thermal winds" in geophysical settings. (3) Radial entropy gradients make the disk gas radially buoyant, but not so buoyant as to directly overcome the angular momentum barrier. In the next three sections we briefly describe what is known about the instabilities arising in these settings.

\subsection{Rossby-Wave Instability}

A bump in the radial pressure profile yields a local extremum in the rotation speed, or more precisely in the vortensity (vorticity divided by surface density). The extremum is linearly unstable to Rossby-wave instability (RWI), which develops into vortices in a few orbits Lovelace et al., 1999, Li et al., 2000, 2001). 
Since the orbital speed's radial gradient is key, the RWI is a special kind of Kelvin-Helmholtz instability found in rotating systems. While the RWI develops under conditions readily studied in numerical simulations (Meheut et al. 2010, 2012b c) the unstable initial state has to be explained in the first place. Suitable conditions can occur near the outer edge of a gap opened in the disk by a planet (Balmforth and Korycansky, 2001. de Val-Borro et al. 2007). In another scenario, a pressure bump forms at a dead zone's inner edge Varnière and Tagger, 2006, Lyra et al. 2009). Varnière used a radially-varying $\alpha$ value for this experiment, while Lyra and Mac Low (2012) performed a global nonideal MHD simulation with radially increasing resistivity and also found a pressure bump, and subsequently a large vortex. In conclusion, the RWI does not make vortices in an unperturbed laminar disk, but requires either planets or neighboring MHD active zones.

\subsection{Goldreich-Schubert-Fricke Instability}

In protostellar disks the temperature and density vary independently. Temperatures are set by starlight heating outside a central accretion-dominated region (Dullemond et al., 2007), while densities are determined by the accretion flow. Surfaces of constant pressure therefore tilt relative to surfaces of constant density, making protostellar disks at least in part baroclinic. The baroclinic regions reach a balance between the gas pressure, gravity and rotational forces by forming a "thermal wind" in which the orbital frequency increases with the distance from the midplane Tassoul, 2007, Fromang et al. 2011). The increase is quadratic where the temperature is constant with height. The resulting shear is locally stable against the KelvinHelmholtz instability (KHI) owing to the vertical density stratification (Rüdiger et al., 2002). KHI can nevertheless occur if the embedded solid particles sediment with respect to the gas, forming a thin midplane layer rotating at Keplerian speed. The shear relative to the slower-rotating gas can yield Richardson numbers low enough for KHI to produce mild local turbulence $(W e i-$ denschilling and Cuzzi, 1993; Johansen et al., 2006a; Barranco, 2009 Lee et al. 2010).

Accretion disks' radial shear also permits an instability first studied for rotating stars' interiors, the Goldreich-Schubert-Fricke (GSF) instability (Goldreich and Schubert, 1967, Fricke, 1968). This can be triggered when the radial shear is Rayleigh stable but receives a boost from vertical shear. Under the combined shear, there exist paths inclined slightly with respect to the vertical, along which the specific angular momentum is constant. Exchanging gas parcels along these paths can be unstable if the disk's vertical stratification is neutrally buoyant, with Brunt-Väisälä frequency zero. Any real, non-zero buoyancy frequency stabilizes the exchange of gas parcels. The instability thus occurs only if the disk either is adiabatically strat- ified, or relaxes thermally on very short time scales. Quick-enough heating and cooling can overcome the stable vertical density stratification. Indeed in the two limiting cases of instantaneous cooling Urpin and Brandenburg, 1998) and isentropic or uniform-entropy stratification (Rüdiger et al. 2002), the buoyancy frequency and thus the Richardson number are effectively zero and the Rayleigh stability criterion generalizes to $\partial_{R} \Omega^{2}(R, z) R^{4}-\left(k_{R} / k_{z}\right) R^{4} \partial_{z} \Omega^{2}(R, z)>0$ Urpin, 2003 Nelson et al. 2013). Hence perturbing the vertical rotation profile can lead to weak, transient turbulence until the shear decays, even in an isothermal flow with an unsheared background state Arlt and Urpin, 2004). When the vertical shear is part of the background profile, the disk undergoes narrow near-vertical overturning motions, leading to sustained accretion stresses at levels $\alpha \sim 10^{-3}$ (Nelson et al. 2013$)$. The instability is suppressed when thermal relaxation enforces an isothermal atmosphere slower than $0.01 \Omega^{-1}$. The threshold is more easily reached when the vertical structure is driven towards isentropy: instability occurs for thermal relaxation times up to $1 \Omega^{-1}$. Candidates for regions with thermodynamics suitable for the GSF are disks with moderate optical depths, and in particular the outer reaches of $\mathrm{T}$ Tauri disks well above the midplane.

\subsection{Baroclinic Vortex Formation}

Vortices can also arise in disks having a radial entropy gradient (Klahr and Bodenheimer, 2003). Unlike the baroclinic instability of stars and planetary atmospheres, which relies on the vertical shear and seems to be suppressed in disks by the radial shear, the baroclinic instability in disks depends on the radial entropy gradient through the Brunt-Väisälä frequency $N^{2}=-\gamma^{-1} \beta_{P} \beta_{S}(H / R)^{2} \Omega^{2}$. This is proportional to the product of the logarithmic gradients in the pressure $P$ and entropy $S=\log \left(P \rho^{-\gamma}\right)$, the latter written as a potential temperature. Here the local pressure scale height $H=c_{s} / \Omega$ and the gradients are $\beta_{P}=d \log P / d \log R$ and $\beta_{S}=\beta_{T}+(1-\gamma) \beta_{\rho}$, with $\beta_{\rho}$ the logarithmic radial density and $\beta_{T}$ the logarithmic radial temperature gradient, all for the vertically unstratified case. Positive $N^{2}$ corresponds to convective stability and negative to unstable stratification if the fluid is non-rotating. In a rotating system the radial motions are affected if the modified Rayleigh criterion $\left(1 / R^{3}\right) \partial_{R}\left[\Omega^{2}(R) R^{4}\right]+N^{2}>0$ holds (a special case of the Solberg-Høiland criterion with rotation; Tassoul, 2007, Chandrasekhar, 1961). Since typically $-N^{2} \ll \Omega^{2}$, all radial convective modes are linearly stable (Klahr, 2004, Johnson and Gammie, 2005, 2006). Yet numerical models indicate vortices preexisting in the disk are amplified if thermal relaxation occurs over approximately the vortices' internal rotation periods (Petersen et al. 2007a b). Because growth requires perturbations of finite amplitude, Lesur and 


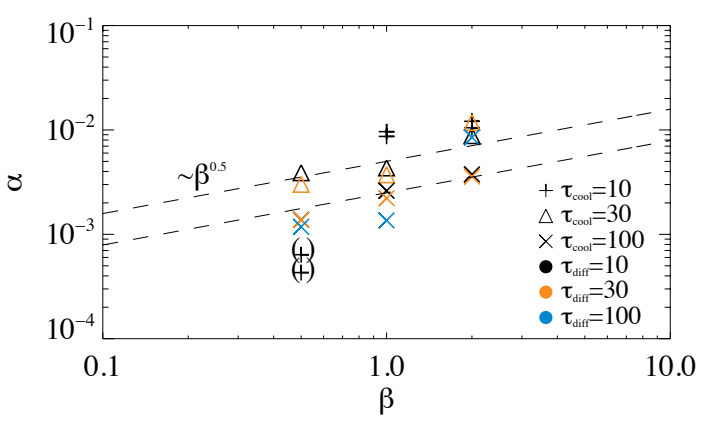

Fig. 5.- Reynolds stress parameter $\alpha$ in saturated 2-D baroclinic turbulence vs. the logarithmic radial gradient in both entropy and pressure, $\beta_{S}=\beta_{P}$. Each calculation is shown by a symbol whose color indicates the timescale for thermal diffusion within the disk plane, and shape indicates the timescale for heating and cooling via the disk surface (Raettig et al., 2013).

Papaloizou (2010) coined the name subcritical baroclinic instability or SBI. The vortex growth rate is proportional to $-\tau N^{2}$, with $\tau$ the thermal relaxation time, provided $\Omega \tau<1$ (Lesur and Papaloizou, 2010, Raettig et al. 2013). The thermal relaxation times needed for SBI are generally $100 \times$ longer than the maximum for the GSF in an isothermal background. SBI thus seems likely to occur in regions of greater optical depth.

Vortices in 3-D are susceptible to the elliptic instability, a parasitic process (Lesur and Papaloizou, 2009). The instability drastically disturbs the flow in vortices rotating fast enough. Rotation is faster in vortices with smaller azimuthal-to-radial aspect ratios, and elliptic instability typically is important at aspect ratios $<4$. More azimuthally-extended vortices may be safe from destruction by the elliptic instability but still internally unstable, and thus turbulent at some level. At least in local 3-D calculations, the parasitic growth does not completely destroy vortices, but weakens them to levels where the elliptic instability is suppressed allowing for new baroclinic growth. The saturated vortices' turbulent transport coefficient $\alpha$ is $0.001-0.01$ depending on the cooling time and entropy gradient (Fig. 5), a range compatible with vortices providing the angular momentum transport behind disks' observed surface density profiles (Andrews et al., 2009, 2010).

\subsection{General Properties of Vortices}

Independently of how they form, vortices extend up to two pressure scale heights in radius and eight or more in azimuth, in both 2- and 3-D calculations. Wider vortices experience a supersonic difference in orbital speed between their inner and outer edges, so a shock truncates the flow pattern. Because the structures are large and can trap solid particles, they might be observable and indeed may already have been ob- served (Brown et al. 2009, Regály et al., 2012, van der Marel et al. 2013).

With magnetic fields well-coupled, vortices are quickly disrupted by a vortex version of the MRI coined the magneto-elliptic instability (Lyra and Klahr, 2011) and occurring only in 3-D calculations. Yet if the disk is magnetically dead this effect should be suppressed.

Like their cousins in the earth's atmosphere, vortices in the body of the disk are not fixed at a radial or azimuthal location, but tend to migrate on reaching a certain amplitude as they exchange angular momentum with their surroundings by emitting spiral waves (Paardekooper et al. 2010). Consequently vortices are amplified by the radial buoyancy, then migrate inward and may disappear into the star or magnetically-active part of the disk. Thus a reliable mechanism is needed for initiating vortices and this is yet to be identified. Possibilities are a RWI relying on planets or other disk turbulence, the spiral density waves launched by the previous generation of vortices, and the pattern generated by the GSF instability. Or, perhaps a further hydrodynamic instability triggers vortex growth. Vortices could create copies of themselves through a nonlinear "critical layer" instability (Marcus et al., 2013). This occurs in disks that are stably-stratified in the vertical direction with little or no thermal relaxation - diametrically opposed to the conditions favoring the GSF. How strict these requirements are and how well they are realized in protostellar disks is still unclear.

In summary we emphasize that no hydrodynamical instability is known to act on a strictly barotropic, vertically unstratified disk without a local extremum in its rotation profile, nor does any known mechanism sustain long-lived vortices within (Shen et al. 2006$)$. However at least two instabilities can operate globally in a baroclinic disk under suitable thermal conditions: the GSF and baroclinic vortex driving instabilities.

Motivated by the terrestrial atmospheric weather patterns arising from individual instabilities driven by solar heating, we have named the total effect of the hydrodynamical instabilities the "disk weather". A key issue is when and where in protostellar disks the heating and cooling timescales support such weather. Calculations to date span only a small fraction of the parameter space. For instance there exists no global, vertically stratified calculation of baroclinic vortices with realistic entropy gradients and radiation transport, owing to the high spatial resolution required. Such calculations will eventually be needed if we are to study the origin, stability and ultimate fate of vortices as weather patterns in protostellar disks.

\section{Observational Signatures and Constraints}

To understand protostellar disks' evolution, we must know which transport processes are at work. The many possible mechanisms mean a reliable answer re- 


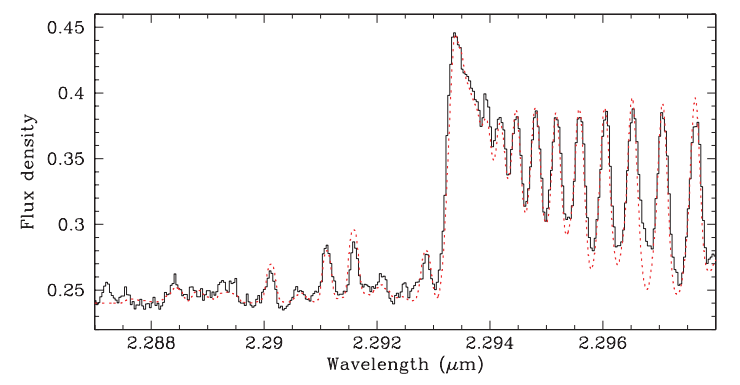

Fig. 6.- Observed 2.3- $\mu \mathrm{m}$ spectrum of young star V1331 Cyg (histogram) overlaid with a model (dotted). The microturbulent broadening used, $4 \mathrm{~km} \mathrm{~s}^{-1}$, exceeds the $0.9-\mathrm{km}_{-} \mathrm{s}^{-1} \mathrm{CO}$ thermal dispersion and is near the sound speed at $2500 \mathrm{~K}$ (Najita et al., 2009).

quires an empirical approach. Observations probe the disks' kinematics, density and temperature structure, ionization state, magnetic fields and composition, each of which carries signatures of the transport processes. While few definitive conclusions can yet be reached, some predictions are now falsifiable. It is worth remembering that the transport process may differ from one disk annulus to the next and from one star to another. For example, gravitational instability is more likely at the higher surface densities found in class I YSOs, while magnetic coupling is easier to achieve at the lower surface densities found in T Tauri disks.

\subsection{Kinematics}

Each transport process has a distinct flow pattern that would serve as a fingerprint if we could only observe with enough spatial and spectral resolution. Disk weather makes vortices, gravitational instability produces spiral shocks, disk winds mean helical outflow and MRI turbulence is transonic near the disk surface.

Non-thermal velocity dispersions in the outer reaches of a few disks have been measured using $\mathrm{mm}$ and sub$\mathrm{mm}$ lines. TW Hya yielded an upper limit $<0.1 c_{s}$, while velocities $\sim 0.5 c_{s}$ were found in the outer disks of HD 163296 (Hughes et al. 2011) and DM Tau (Guilloteau et al. 2012).

Velocities nearer the star are also detected using infrared (IR) lines formed in the disk atmosphere. The evidence again is for turbulent speeds comparable to the sound speed (Carr et al. 2004; Hartmann et al. 2004: Najita et al., 2009). In Fig. 6, the disk's rotation and microturbulence are jointly constrained by the widths of the isolated water and $\mathrm{CO}$ lines, the $\mathrm{CO}$ lines' beating immediately redward of the bandhead, and their strengths far from the bandhead compared to the bandhead itself.

Both MRI turbulence (Hawley et al., 1995, Miller and Stone, 2000, Simon et al., 2011b) and gravitoturbulence (Forgan et al. $\mid 2012)$ have anisotropies that could distinguish them from other transport processes.
The velocity dispersion ellipsoid is best accessed using saturated emission lines, especially those of heavy species for which the thermal speed is less than the turbulent speed (Horne, 1995).

Warm disk outflows inferred from atomic oxygen emission line profiles in the optical (Rigliaco et al. 2013) could arise either in a photoevaporative wind (chapter by Alexander et al.) or in a magnetocentrifugal wind. If a cold outflow were observed, it would uniquely indicate magnetic acceleration.

\subsection{Structure in the Gas and Dust}

Mapping surface densities at $\mathrm{mm}$ and sub-mm wavelengths (Andrews et al. 2009 , Isella et al. $2009, A n$ drews et al. , 2010) offers the potential to determine both whether transport mechanisms can operate and whether they are operating. Questions of the first kind include whether the mass column is low enough for ionizing radiation to reach the midplane and whether the Toomre Q parameter is low enough for gravitational instability. A question of the second kind, now accessible with ALMA, is whether any spiral features more closely resemble the density waves raised by self-gravity Cossins et al. 2009, 2010) or those excited in MRI turbulence (Heinemann and Papaloizou, 2009, 2012).

Millimeter mapping also enables detection of vortices. Modeling indicates vortices trap solid particles (Inaba and Barge, 2006, Lyra et al., 2008, 2009, Meheut et al.. 2012a), and the continuum emission from $\mathrm{mm}$-sized grains shows big asymmetries in several cases (Brown et al. 2009). Among the most spectacular is a crescent feature observed at wavelength $0.44 \mathrm{~mm}$ near the inner rim of the transitional disk around Oph IRS 48 (van der Marel et al. 2013$)$. The feature is $>100 \times$ brighter than emission on the opposite side of the star, while no such asymmetry appears either in the mid-IR emission from $\mu$ m-sized dust, or in the line emission from CO gas. Developing such a strong feature in the particles appears to require a long-lived asymmetry in the gas (Birnstiel et al. 2013 . Ataiee et al. 2013, Lyra and Lin, 2013). Along similar lines, zonal flows will be detectable by axisymmetric rings and gaps (Ruge et al. 2013). Care is needed to distinguish gaps made by different processes.

Young stars' IR spectral energy distributions (SEDs) and scattered light imaging sample the distribution of sub- $\mu \mathrm{m}$ grains. Turbulence of some kind appears to be needed to keep enough of the dust suspended in the disk atmospheres to account for the reprocessed and scattered starlight (Fig. 7) Watson et al., 2007, Dullemond and Dominik, 2005). Typical SEDs of diskbearing young stars can be explained using dust stirred by turbulence at $1 \%$ of the sound speed, almost independent of stellar mass over the range from 0.08 to $2 M_{\odot}$ (Mulders and Dominik, 2012). Higher turbulent speeds are allowed if balanced by reduced dust abundances, or reduced gas masses. 

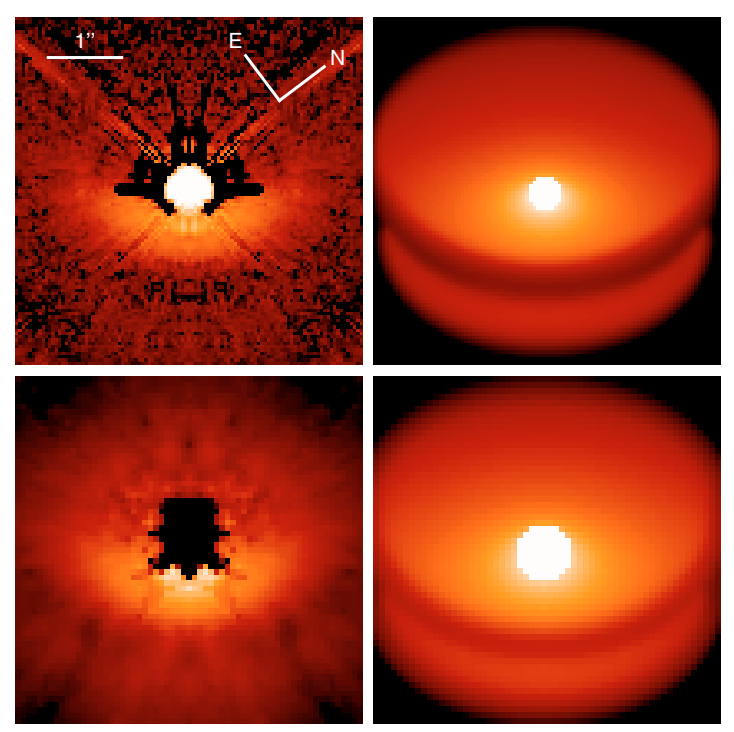

Fig. 7.- Images of the disk around IM Lupi at wavelengths 0.6 (top) and $1.6 \mu \mathrm{m}$ (bottom) from Hubble (left panels), alongside models fitted jointly to the images and optical-to-mm-wavelength SED (right). While $\mathrm{mm}$ data show substantial grain growth and settling, these scattered light images' brightness indicates sub- $\mu \mathrm{m}$ grains remain suspended in the disk atmosphere, requiring stirring (Pinte et al., 2008).

Dust evolution is coupled intimately to turbulence driven by magnetic forces, because recombination on grains can reduce the gas conductivity below the threshold for MRI turbulence (Sano et al. 2000, Ilgner and Nelson, 2006a; Wardle, 2007). In the absence of turbulence, grains grow and settle within a fraction of the disk lifetime. On the other hand, turbulent stirring enhances solid particles' collision rates, speeding growth if the collisions are slow and causing fragmentation if the collisions are fast (Brauer et al., 2008. Zsom et al. 2010: Birnstiel et al. 2010). It remains to be seen whether the mutual coupling can account for the correlation observed between grain size and settling (Sargent et al. 2009$)$ and the lack of correlation of grain size with other parameters.

Further information on transport processes is contained in the disk thickness. MRI turbulence leads to the formation of a magnetically-supported disk atmosphere that is optically-thin to its own thermal emission, because good magnetic coupling requires recombination that is not too fast, and the opacity, like the recombination, depends on the dust cross-section $(B a i$ and Goodman, 2009). At the same time the magnetized atmosphere is optically-thick to the starlight entering at a grazing angle (Hirose and Turner, 2011). The disk thickness then reveals the magnetic field strengths rather than the internal temperatures. A contrary effect comes from magnetic fields generated in a wind with midplane plasma beta near unity. These can compress the disk so that its thickness is less than the hydrostatic value (Königl et al., 2010).

Variability in the IR excess is widespread among young stars with disks, having amplitudes 0.1-0.5 magnitude and timescales as short as a week Luhman et al., 2008, Muzerolle et al., 2009, Luhman et al. 2010 Flaherty et al. , 2011). In some cases the variations correlate with markers of the star's accretion and magnetic activity (Flaherty et al., 2013$)$ while in others they do not (Morales-Calderón et al., 2011). The widespread occurrence of the variability suggests a universal process, such as interaction with the stellar magnetosphere in the first situation and turbulencedriven changes in the size or shape of the starlightreprocessing disk material in the second.

\subsection{Temperature Profiles}

While accretion flows by their very nature involve the release of gravitational potential energy, the transport processes described above differ in where the power is deposited. A variety of temperature profiles may result. Since spectral lines sample the temperature gradients at the depths where they form, spectroscopy could be used to constrain which mechanisms are at work, even where it is not feasible to reach spectral resolutions high enough to measure kinematics.

The accretion power per unit disk area falls off with distance from the star as $r^{-3}$ approximately, a steeper dependence than the illuminating starlight, so that with increasing mass flow rate the accretion power becomes dominant first near the star. The accretion power determines the midplane temperature within $6 \mathrm{AU}$ and the surface temperature within $2 \mathrm{AU}$ of an $0.9 M_{\odot} \mathrm{T}$ Tauri star accreting at $10^{-7} M_{\odot} \mathrm{yr}^{-1}$ with the heating distributed in proportion to the mass (Dullemond et al. 2007). Thus the signatures of accretion heating are most likely to appear in spectral lines formed within a few $\mathrm{AU}$ of the star.

A well-established example is the $\mathrm{CO}$ rovibrational first overtone band, which arises in the disk atmosphere within $1 \mathrm{AU}$ of the star and can be either in emission, when the atmosphere is hotter than the interior due to stellar illumination, or in absorption, when the internal heating is strong enough to dominate (Calvet et al. 1991, Najita et al., 2007; Connelley and Greene, 2010). The CO rovibrational bands' power source can be the accretion flow if the associated heating corresponds to a stress approaching the pressure in the disk atmosphere (Glassgold et al. 2004). Stellar X-ray heating as the power source is insufficient.

MRI turbulence yields dissipation distributed throughout the disk thickness when the gas is fully-ionized (Flaig et al. 2010, 2012). The dissipation occurs higher on average than the accretion flow, owing to the magnetic fields' buoyancy, and is of course further concentrated toward the surface if the interior is too 
weakly-ionized to couple to the magnetic fields. Consequently the midplane is cooler than if the dissipation were distributed uniformly in the column (Hirose and Turner, 2011). The MRI turbulent layer emits much of its power in spectral lines. Line surface brightnesses might one day reveal the mass flow rate as a function of distance from the star (Bai and Goodman, 2009).

\subsection{Magnetic Field Measurements}

To distinguish the two magnetic transport mechanisms - MRI turbulence and magnetocentrifugal winds - it would be valuable to know how the fields are oriented and whether they are straight or tangled. Grains aligned by toroidal magnetic fields in the outer parts of T Tauri disks ought to detectably polarize the thermal emission (Cho and Lazarian, 2007) but the sub-mm flux from scales $>100$ AU is weakly polarized in the five bright protostellar disks examined so far, with $\mathrm{P}<1 \%$ (Hughes et al. $, 2009,2013)$. The lack of polarization could be due to magnetic fields that are either tangled or, for systems viewed face-on, poloidal rather than toroidal. Magnetized turbulence and winds thus cannot yet be separated. Better sub-mm spatial resolution and far-IR polarimeters will enable similar tests to be applied to material nearer the star.

A record of the magnetic fields in the protosolar disk was once thought to be preserved in the remanent magnetization of chondritic meteorites last melted during the planet-forming epoch. The remanence now appears more likely to sample the magnetic fields in the unmelted chondritic surface layer of a partiallydifferentiated planetesimal that sustained dynamo action in its convecting metallic core (Weiss et al. 2010).

\subsection{Detecting Transport Through its Effects on Composition}

Departures from local chemical equilibrium can indicate material being transported along gradients, especially gradients in temperature and radiation intensity. A classic case is the presence of crystalline silicate grains in comets, which because of their icy makeup surely formed beyond the Solar nebula's snow line. Grains in the interstellar medium are almost entirely amorphous (Kemper et al. 2005), and crystalline structure is destroyed by cosmic rays within 70 Myr Bringa et al., 2007). The comet grains' crystallinity thus most likely arose by heating to $1000 \mathrm{~K}$ or more within the Solar nebula. Crystalline silicate grains are detected in comets through IR spectroscopy (Min et al. 2005 , Lisse et al. 2006) and found in the sample brought back from Comet $81 \mathrm{P} /$ Wild 2 by the Stardust mission. The returned sample shows a wide range of olivine and pyroxene compositions. Among the grains is a refractory, CAI-like particle processed at temperatures above $2000 \mathrm{~K}$ (Zolensky et al., 2006). Taken together, these properties appear to require large-scale radial trans- port in the protosolar disk.

Crystalline silicates also occur in the disks around other young stars. The silicate grains' crystalline fraction is similar across the range of annuli in $\mathrm{T}$ Tauri disks producing the $10-33 \mu \mathrm{m}$ IR bands Sargent et al. 2009), indicating either a dispersed source or efficient transport. Variations in the $10-$ and $20-\mu \mathrm{m}$ signatures of crystalline forsterite during an outburst of EX Lupi suggest supersonic transport, compatible with grains dragged along by a wind (Juhász et al., 2012$)$.

A new kind of probe of the transport is the mid-IR emission from water and organic molecules discovered in the atmospheres of many T Tauri disks (Salyk et al. 2008, Carr and Najita, 2008, Pontoppidan et al., 2010. Salyk et al. , 2011; Carr and Najita, 2011). In particular, there is a trend between the $\mathrm{HCN}$-to-water ratio at $\mathrm{AU}$ distances and the sub-mm continuum flux outside 20 AU, suggested to arise from water ice accumulating into solid bodies (Najita et al. 2013).

Disks' compositions can be easier to measure than their kinematics, but carry fewer clear signatures of specific transport processes since the details of the flow often matter less than whether the material has been exposed to high temperatures or energetic radiation. Nevertheless the transport processes discussed in this chapter have some distinctive features with the potential to yield differing chemical signatures:

MRI turbulence yields mixing and angular momentum transfer coefficients roughly equal (Johansen and Klahr, 2005, Turner et al., 2006, Fromang and Papaloizou, 2006) with the angular momentum transfer increasing faster than the mixing as the background vertical magnetic field is made stronger Johansen et al. 2006b). As discussed in \$2 the magnetic activity at planet-forming distances is strongest in the disk surface layers. The global meridional circulation predicted in simple viscous models has not so far been seen in MHD calculations (Fromang et al. 2011, Flock et al. 2011).

Magnetocentrifugal winds drive the gas radially inward near the midplane and outward above the height where the inclined field lines' azimuthal speed reaches Keplerian, in radially-localized stationary solutions (Salmeron et al., 2011 ). Rapid inward and outward radial transport near the surface of a wind-driving disk could also come from episodic channel flows (Suzuki and Inutsuka, 2009) whose repeated breakup would cause some vertical mixing.

Gravitoturbulence can redistribute material in radius as rapidly as can MRI turbulence Michael et al. 2012). Even so a contaminant may remain inhomogeneous during the global mixing timescale if the unstable features are bigger than the disk thickness Boss. 2007a, 2013) or if shock passages affect desorption from 
grains or destruction in chemical reactions (Ilee et al. 2011). The shock heating associated with gravitational instability appears to drive little convection in the vertical direction when an accurate boundary condition is applied at the disk photosphere (Cai et al., 2010).

Several attempts have been made to bring these ideas about transport into contact with composition measurements. Crystalline grains can be moved through the disk to the comet-forming region with either a sustained radially-outward midplane flow, or a diffusivity for turbulent mixing exceeding that for angular momentum transfer Hughes and Armitage, 2010). Vertical mixing with a coefficient in the range inferred for the radial angular momentum transfer enhances the abundances of some carbon- and sulfurbearing molecules in $\mathrm{T}$ Tauri disks by orders of magnitude, by bringing reactive atoms and ions from the overlying photodissociation layers into more shielded molecular regions (Willacy et al., 2006, Semenov and Wiebe, 2011). The molecular abundances are affected more by turbulent mixing than by a steady-state disk wind (Heinzeller et al. 2011). Comparing these models with the molecular column densities obtained from $\mathrm{mm}$ line measurements, mostly near and outside $100 \mathrm{AU}$, yields mixed results suggesting further complexities in the chemistry, dynamics or both.

\section{Summary and Outlook}

Magneto-rotational turbulence is suppressed by ambipolar diffusion in local stratified models of $\mathrm{T}$ Tauri disks' planet-forming regions, even when the Elsasser number and plasma beta criteria indicate turbulence in a surface layer about one scale height thick $(B a i$ and Stone, 2013b). Furthermore the turbulence is suppressed by a strong Hall effect in unstratified calculations (Kunz and Lesur, 2013). These results raise serious doubts about whether MRI turbulence is effective at 1-10 AU even in surface layers. Further progress might involve (1) bringing the temperatures in MHD calculations up to the level of sophistication found in thermo-chemical models, where stellar X-ray and UV photons heat the disk atmosphere to $>2000 \mathrm{~K}$ (Glassgold et al., 2004, Ercolano et al., 2008; Owen et al., 2010: Aresu et al. 2011; Akimkin et al. 2013), surely affecting the conductivity, and (2) allowing the Ohmic, Hall and ambipolar terms all to vary with height. No non-linear MHD calculation yet treats this full problem. MRI turbulence likely remains important in the thermally-ionized gas inside $1 \mathrm{AU}$ and the less-dense material beyond $10 \mathrm{AU}$. A key unresolved issue here is the saturation amplitudes at realistic Prandtl numbers.

Magnetocentrifugal disk winds appear to be favored in disks with the interior dominated by Ohmic diffusion and the surface dominated by ambipolar diffusion, threaded by magnetic fields that are not too weak.
Disk winds and MRI turbulence coexist if the magnetic fields are weak enough that the MRI wavelength fits in the disk thickness. The dependences of both MRI turbulence and magnetocentrifugal winds on the net field point to the large-scale magnetic flux transport as a major issue that must be adequately dealt if we are to understand protostellar disks' long-term evolution.

Gravitational instability occurs in disks whose mass ratios with their central stars exceed their aspect ratios. It is most easily triggered in the outer reaches, since $c_{s} \Omega$ falls off faster with radius than the typical surface density profile. Gravitational instability can be important during the protostellar phase when the disk is fed rapidly with material falling in from the surrounding envelope. The outcome of the instability depends on how quickly the compressed gas cools, and room remains for dynamical modeling with more complete treatment of the radiative losses.

All the disk weather processes discussed here, including Rossby and baroclinic vortices and the Goldreich-Schubert-Fricke instability, depend on radiative forcing for their energy supply. There is an urgent need for calculations with better heating and cooling. The instabilities grow slower than the MRI and so can reach significant amplitudes only in regions lacking MRI turbulence. Whether they survive in the presence of a magnetically-launched wind is unclear.

While all the transport processes we discuss are commonly measured and compared using the ShakuraSunyaev $\alpha$-parameter, none behaves like the simple Shakura and Sunyaev (1973) model. MRI turbulent stresses fall off with height slower than the gas pressure, even when the magnetic coupling is good throughout. Magnetocentrifugal winds expel the angular momentum vertically rather than radially. The energy escapes in a Poynting flux that dissipates far away, leaving the disk unheated. Gravitational instability leads to intermittent shock dissipation, and disk weather yields transport localized around vortices. Any treatment of these processes using an $\alpha$-model should therefore be evaluated carefully.

Observations offer tantalizing clues to the transport processes. Non-thermal motions are detected, but the spatial resolution is too coarse to unequivocally distinguish turbulence from winds or vortices. In order of decreasing distance from the central star,

- Millimeter lines formed outside $100 \mathrm{AU}$ in two disks show transonic superthermal widths (Hughes et al., 2011; Guilloteau et al., 2012). These are explained most easily using turbulence but could also arise from outflows.

- Keeping enough dust suspended in the atmosphere to explain both the scattered starlight at $\sim 50 \mathrm{AU}$ and the SEDs requires some kind of stirring. MRI turbulence can do the job where magnetic coupling is good. Outflows and vor- 
tices could potentially also reproduce the dust distributions, but better models are called for.

- Asymmetric sub-mm surface brightness maps in a few disks (van der Marel et al. 2013) have so far been reproduced only using long-lived nonaxisymmetric gas density disturbances. Vortices are the most viable explanation.

- Applying angular momentum constraints to jets yields launch points within a few AU of the stars (Anderson et al., 2003, Coffey et al., 2007). Disks' inner regions clearly launch outflows.

- Superthermal CO overtone linewidths indicate transonic turbulence in the atmosphere inside 1 AU, where the gas is thermally ionized, consistent with MRI Carr et al. 2004, Hartmann et al., 2004, Najita et al. | 2009). On the other hand, the blueshifted central peaks in many young stars' CO fundamental band suggest outflows (Brown et al. 2013). The two kinds of flows likely occur together in some disks.

A key to further progress in understanding protostellar disk evolution is to bring the models closer to observations like these, seeking clearer signatures of the proposed transport processes.

NT was supported at the Jet Propulsion Laboratory, California Institute of Technology by NASA grant 11-OSS11-0074; SF by the European Research Council under the E.U. Seventh Framework Programme (FP7/2007-2013) / ERC Grant agreement 258729; HK by the Deutsche Forschungsgemeinschaft Schwerpunktprogramm (DFG SPP) 1385 "The first ten million years of the solar system"; GL by the European Community via contract PCIG09-GA-2011294110; and MW by Australian Research Council grant DP120101792.

\section{REFERENCES}

Akimkin V. et al. (2013) Astrophys. J., 766, 8. Anderson J. M. et al. (2003) Astrophys. J. Lett., 590, L107. Andrews S. M. et al. (2009) Astrophys. J., 700, 1502. Andrews S. M. et al. (2010) Astrophys. J., 723, 1241. Aresu G. et al. (2011) Astron. Astrophys., 526, A163. Arlt R. and Urpin V. (2004) Astron. Astrophys., 426, 755. Ataiee S. et al. (2013) Astron. Astrophys., 553, L3.

Avila M. (2012) Physical Review Letters, 108, 12, 124501.

Bai X.-N. (2011a) Astrophys. J., 739, 50.

Bai X.-N. (2011b) Astrophys. J., 739, 51.

Bai X.-N. (2013) Astrophys. J., 772, 96.

Bai X.-N. and Goodman J. (2009) Astrophys. J., 701, 737. Bai X.-N. and Stone J. M. (2011) Astrophys. J., 736, 144. Bai X.-N. and Stone J. M. (2013a) Astrophys. J., 767, 30. Bai X.-N. and Stone J. M. (2013b) Astrophys. J., 769, 76. Balbus S. A. and Hawley J. F. (1991) Astrophys. J., 376, 214.
Balbus S. A. and Henri P. (2008) Astrophys. J., 674, 408.

Balbus S. A. and Papaloizou J. C. B. (1999) Astrophys. J., 521, 650 .

Balmforth N. J. and Korycansky D. G. (2001) Mon. Not. R. Astron. Soc., 326, 833.

Barge P. and Sommeria J. (1995) Astron. Astrophys., 295, L1.

Barranco J. A. (2009) Astrophys. J., 691, 907.

Beckwith K. et al. (2009) Astrophys. J., 707, 428.

Beckwith K. et al. (2011) Mon. Not. R. Astron. Soc., 416, 361.

Bergin E. A. et al. (2007) Protostars and Planets V, pp. 751-766.

Bethell T. J. and Bergin E. A. (2011) Astrophys. J., 739, 78 .

Birnstiel T. et al. (2010) Astron. Astrophys., 513, A79.

Birnstiel T. et al. (2013) Astron. Astrophys., 550, L8.

Blaes O. M. and Balbus S. A. (1994) Astrophys. J., 421, 163.

Blandford R. D. and Payne D. G. (1982) Mon. Not. R. Astron. Soc., 199, 883.

Bodo G. et al. (2008) Astron. Astrophys., 487, 1.

Bodo G. et al. (2011) Astrophys. J., 739, 82.

Boley A. C. (2009) Astrophys. J. Lett., 695, L53.

Boss A. P. (1997) Science, 276, 1836.

Boss A. P. (2007a) Astrophys. J., 660, 1707.

Boss A. P. (2007b) Astrophys. J. Lett., 661, L73.

Boss A. P. (2013) Astrophys. J., 773, 5.

Brandenburg A. et al. (1995) Astrophys. J., 446, 741.

Brauer F. et al. (2008) Astron. Astrophys., 480, 859.

Bringa E. M. et al. (2007) Astrophys. J., 662, 372.

Brown J. M. et al. (2009) Astrophys. J., 704, 496.

Brown J. M. et al. (2013) Astrophys. J., 770, 94.

Cai K. et al. (2008) Astrophys. J., 673, 1138.

Cai K. et al. (2010) Astrophys. J. Lett., 716, L176.

Calvet N. et al. (1991) Astrophys. J., 380, 617.

Cao X. and Spruit H. C. (2002) Astron. Astrophys., 385, 289.

Carr J. S. and Najita J. R. (2008) Science, 319, 1504.

Carr J. S. and Najita J. R. (2011) Astrophys. J., 733, 102.

Carr J. S. et al. (2004) Astrophys. J., 603, 213.

Chandrasekhar S. (1960) Proceedings of the National Academy of Science, 46, 253.

Chandrasekhar S. (1961) Hydrodynamic and hydromagnetic stability, Dover Publications; Dover edition (February 1, 1981).

Cho J. and Lazarian A. (2007) Astrophys. J., 669, 1085.

Ciesla F. J. and Cuzzi J. N. (2006) Icarus, 181, 178.

Clarke C. J. et al. (2007) Mon. Not. R. Astron. Soc., 381, 1543.

Cleeves L. I. et al. (2013) Astrophys. J., 772, 5.

Coffey D. et al. (2007) Astrophys. J., 663, 350.

Connelley M. S. and Greene T. P. (2010) Astron. J., 140, 1214.

Cossins P. et al. (2009) Mon. Not. R. Astron. Soc., 393, 1157.

Cossins P. et al. (2010) Mon. Not. R. Astron. Soc., 40\%, 181.

Davis S. W. et al. (2010) Astrophys. J., 713, 52.

de Val-Borro M. et al. (2007) Astron. Astrophys., 471, 1043.

Desch S. J. (2004) Astrophys. J., 608, 509.

Desch S. J. et al. (2004) Astrophys. J., 602, 528. 
Dittrich K. et al. (2013) Astrophys. J., 763, 117.

Douglas T. A. et al. (2013) Mon. Not. R. Astron. Soc., 433, 2064.

Dullemond C. P. and Dominik C. (2005) Astron. Astrophys., 434, 971.

Dullemond C. P. et al. (2007) Protostars and Planets V, pp. $555-572$.

Dzyurkevich N. et al. (2010) Astron. Astrophys., 515, A70.

Dzyurkevich N. et al. (2013) Astrophys. J., 765, 114.

Ercolano B. et al. (2008) Astrophys. J., 688, 398.

Ferreira J. (1997) Astron. Astrophys., 319, 340.

Ferreira J. and Pelletier G. (1993) Astron. Astrophys., 276, 625.

Ferreira J. and Pelletier G. (1995) Astron. Astrophys., 295, 807.

Ferro-Fontán C. and Gómez de Castro A. I. (2003) Mon. Not. R. Astron. Soc., 342, 427.

Flaherty K. M. et al. (2011) Astrophys. J., 732, 83.

Flaherty K. M. et al. (2013) Astron. J., 145, 66.

Flaig M. et al. (2010) Mon. Not. R. Astron. Soc., 409, 1297.

Flaig M. et al. (2012) Mon. Not. R. Astron. Soc., 420, 2419.

Fleming T. and Stone J. M. (2003) Astrophys. J., 585, 908.

Flock M. et al. (2011) Astrophys. J., 735, 122.

Flock M. et al. (2012) Astrophys. J., 744, 144.

Forgan D. and Rice K. (2013) Mon. Not. R. Astron. Soc., 430, 2082.

Forgan D. et al. (2011) Mon. Not. R. Astron. Soc., 410, 994.

Forgan D. et al. (2012) Mon. Not. R. Astron. Soc., 426, 2419.

Fricke K. (1968) Z. Astrophys., 68, 317.

Fromang S. (2005) Astron. Astrophys., 441, 1.

Fromang S. and Nelson R. P. (2006) Astron. Astrophys., 457, 343.

Fromang S. and Nelson R. P. (2009) Astron. Astrophys., 496, 597.

Fromang S. and Papaloizou J. (2006) Astron. Astrophys., 452, 751.

Fromang S. and Papaloizou J. (2007) Astron. Astrophys., $476,1113$.

Fromang S. et al. (2007) Astron. Astrophys., 476, 1123.

Fromang S. et al. (2011) Astron. Astrophys., 534, A107.

Fromang S. et al. (2013) Astron. Astrophys., 552, A71.

Gammie C. F. (1996) Astrophys. J., 457, 355.

Gammie C. F. (2001) Astrophys. J., 553, 174.

Garmire G. et al. (2000) Astron. J., 120, 1426.

Glassgold A. E. et al. (2004) Astrophys. J., 615, 972.

Gnedin O. Y. et al. (1995) Astron. J., 110, 1105.

Goldreich P. and Lynden-Bell D. (1965) Mon. Not. R. Astron. Soc., 130, 125.

Goldreich P. and Schubert G. (1967) Astrophys. J., 150, 571.

Gressel O. (2010) Mon. Not. R. Astron. Soc., 405, 41.

Gressel O. et al. (2011) Mon. Not. R. Astron. Soc., 415, 3291.

Guan X. et al. (2009) Astrophys. J., 694, 1010.

Guilloteau S. et al. (2012) Astron. Astrophys., 548, A70.

Harsono D. et al. (2011) Mon. Not. R. Astron. Soc., 413, 423.

Hartmann L. et al. (2004) Astrophys. J., 609, 906.

Hawley J. F. and Balbus S. A. (1992) Astrophys. J., 400, 595.
Hawley J. F. and Stone J. M. (1995) Computer Physics Communications, 89, 127.

Hawley J. F. and Stone J. M. (1998) Astrophys. J., 501, 758.

Hawley J. F. et al. (1995) Astrophys. J., 440, 742.

Hawley J. F. et al. (1996) Astrophys. J., 464, 690.

Hawley J. F. et al. (2013) Astrophys. J., 772, 102.

Heinemann T. and Papaloizou J. C. B. (2009) Mon. Not. R. Astron. Soc., 397, 64.

Heinemann T. and Papaloizou J. C. B. (2012) Mon. Not. R. Astron. Soc., 419, 1085.

Heinzeller D. et al. (2011) Astrophys. J., 731, 115.

Herault J. et al. (2011) Physical Review E, 84, 3, 36321.

Hirose S. and Turner N. J. (2011) Astrophys. J. Lett., 732, L30.

Hopkins P. F. and Christiansen J. L. (2013) Astrophys. J., 776, 48.

Horne K. (1995) Astron. Astrophys., 297, 273.

Hughes A. L. H. and Armitage P. J. (2010) Astrophys. J., 719, 1633.

Hughes A. M. et al. (2009) Astrophys. J., 704, 1204.

Hughes A. M. et al. (2011) Astrophys. J., 727, 85.

Hughes A. M. et al. (2013) Astron. J., 145, 115.

Igea J. and Glassgold A. E. (1999) Astrophys. J., 518, 848.

Ilee J. D. et al. (2011) Mon. Not. R. Astron. Soc., 417, 2950.

Ilgner M. and Nelson R. P. (2006a) Astron. Astrophys., 445, 205.

Ilgner M. and Nelson R. P. (2006b) Astron. Astrophys., 445, 223.

Ilgner M. and Nelson R. P. (2008) Astron. Astrophys., 483, 815.

Inaba S. and Barge P. (2006) Astrophys. J., 649, 415.

Isella A. et al. (2009) Astrophys. J., 701, 260.

Ji H. et al. (2006) Nature, 444, 343.

Jin L. (1996) Astrophys. J., 457, 798.

Johansen A. and Klahr H. (2005) Astrophys. J., 634, 1353.

Johansen A. et al. (2006a) Astrophys. J., 643, 1219.

Johansen A. et al. (2006b) Mon. Not. R. Astron. Soc., 370, L71.

Johansen A. et al. (2009) Astrophys. J., 697, 1269.

Johnson B. M. and Gammie C. F. (2003) Astrophys. J., $597,131$.

Johnson B. M. and Gammie C. F. (2005) Astrophys. J., 626, 978.

Johnson B. M. and Gammie C. F. (2006) Astrophys. J., 636, 63.

Johnson E. T. et al. (2006) Astrophys. J., 647, 1413.

Juhász A. et al. (2012) Astrophys. J., 744, 118.

Julian W. H. and Toomre A. (1966) Astrophys. J., 146, 810.

Kemper F. et al. (2005) Astrophys. J., 633, 534.

Kim W.-T. and Ostriker E. C. (2000) Astrophys. J., 540, 372.

Kitchatinov L. L. and Rüdiger G. (2010) Astron. Astrophys., 513, L1.

Klahr H. (2004) Astrophys. J., 606, 1070.

Klahr H. (2007) in: IAU Symposium, vol. 239, (edited by F. Kupka, I. Roxburgh, and K. L. Chan), pp. 405-416.

Klahr H. and Bodenheimer P. (2006) Astrophys. J., 639, 432.

Klahr H. H. and Bodenheimer P. (2003) Astrophys. J., 582, 869. 
Königl A. et al. (2010) Mon. Not. R. Astron. Soc., 401, 1, 479.

Königl A. et al. (2010) Mon. Not. R. Astron. Soc., 401, 479.

Kratter K. M. et al. (2010) Astrophys. J., 708, 1585.

Krumholz M. R. et al. (2007) Astrophys. J., 656, 959.

Kunz M. W. and Balbus S. A. (2004) Mon. Not. R. Astron. Soc., 348, 355.

Kunz M. W. and Lesur G. (2013) Mon. Not. R. Astron. Soc., 434, 2295.

Lathrop D. P. et al. (1992) Physical Review Letters, 68, 1515.

Latter H. N. et al. (2010) Mon. Not. R. Astron. Soc., 406, 848.

Lee A. T. et al. (2010) Astrophys. J., 718, 1367.

Lesur G. and Longaretti P.-Y. (2005) Astron. Astrophys., $444,25$.

Lesur G. and Longaretti P.-Y. (2007) Mon. Not. R. Astron. Soc., 378, 1471.

Lesur G. and Longaretti P.-Y. (2009) Astron. Astrophys., $504,309$.

Lesur G. and Ogilvie G. I. (2010) Mon. Not. R. Astron. Soc., 404, L64.

Lesur G. and Papaloizou J. C. B. (2009) Astron. Astrophys., 498,1 .

Lesur G. and Papaloizou J. C. B. (2010) Astron. Astrophys., $513, \mathrm{~A} 60+$.

Lesur G. et al. (2013) Astron. Astrophys., 550, A61.

Li H. et al. (2000) Astrophys. J., 533, 1023.

Li H. et al. (2001) Astrophys. J., 551, 874.

Lin D. N. C. and Papaloizou J. C. B. (1993) in: Protostars and Planets III, (edited by E. H. Levy and J. I. Lunine), pp. $749-835$.

Lisse C. M. et al. (2006) Science, 313, 635.

Lodato G. and Rice W. K. M. (2004) Mon. Not. R. Astron. Soc., 351, 630.

Lodato G. and Rice W. K. M. (2005) Mon. Not. R. Astron. Soc., 358, 1489.

Longaretti P.-Y. and Lesur G. (2010) Astron. Astrophys., 516, A51.

Lovelace R. V. E. et al. (1999) Astrophys. J., 513, 805.

Lubow S. H. and Spruit H. C. (1995) Astrophys. J., 445, 337.

Lubow S. H. et al. (1994a) Mon. Not. R. Astron. Soc., 267, 235.

Lubow S. H. et al. (1994b) Mon. Not. R. Astron. Soc., 268, 1010.

Luhman K. L. et al. (2008) Astrophys. J., 675, 1375.

Luhman K. L. et al. (2010) Astrophys. J. Suppl., 186, 111.

Lyra W. and Klahr H. (2011) Astron. Astrophys., 527, A138.

Lyra W. and Lin M.-K. (2013) Astrophys. J., 775, 17.

Lyra W. and Mac Low M.-M. (2012) Astrophys. J., 756, 62.

Lyra W. et al. (2008) Astron. Astrophys., 491, L41.

Lyra W. et al. (2009) Astron. Astrophys., 497, 869.

Mac Low M.-M. et al. (1995) Astrophys. J., 442, 726.

Mamatsashvili G. R. and Rice W. K. M. (2010) Mon. Not. R. Astron. Soc., 406, 2050.

Marcus P. S. et al. (2013) Physical Review Letters, 111, 8, 084501.

Matzner C. D. and Levin Y. (2005) Astrophys. J., 628, 817. Meheut H. et al. (2010) Astron. Astrophys., 516, A31.
Meheut H. et al. (2012a) Astron. Astrophys., 545, A134.

Meheut H. et al. (2012b) Astron. Astrophys., 542, A9.

Meheut H. et al. (2012c) Mon. Not. R. Astron. Soc., 422, 2399.

Meru F. and Bate M. R. (2011) Mon. Not. R. Astron. Soc., 411, L1.

Meru F. and Bate M. R. (2012) Mon. Not. R. Astron. Soc., 427, 2022.

Meschiari S. and Laughlin G. (2008) Astrophys. J. Lett., 679, L135.

Michael S. et al. (2011) Astrophys. J. Lett., 737, L42.

Michael S. et al. (2012) Astrophys. J., 746, 98.

Mignone A. et al. (2007) Astrophys. J. Suppl., 170, 228.

Miller K. A. and Stone J. M. (2000) Astrophys. J., 534, 398.

Min M. et al. (2005) Icarus, 179, 158.

Mohanty S. et al. (2013) Astrophys. J., 764, 65.

Moll R. (2012) Astron. Astrophys., 548, A76.

Morales-Calderón M. et al. (2011) Astrophys. J., 733, 50.

Mulders G. D. and Dominik C. (2012) Astron. Astrophys., 539, A9.

Müller T. W. A. et al. (2012) Astron. Astrophys., 541, A123.

Murphy G. C. et al. (2010) Astron. Astrophys., 512, A82.

Muzerolle J. et al. (2009) Astrophys. J. Lett., 704, L15.

Najita J. R. et al. (2007) Protostars and Planets V, pp. 507-522.

Najita J. R. et al. (2009) Astrophys. J., 691, 738.

Najita J. R. et al. (2013) Astrophys. J., 766, 134.

Nelson R. P. and Papaloizou J. C. B. (2004) Mon. Not. R. Astron. Soc., 350, 849.

Nelson R. P. et al. (2013) Mon. Not. R. Astron. Soc., 435, 2610 .

Ogilvie G. I. (2012) Mon. Not. R. Astron. Soc., 423, 1318.

Oishi J. S. and Mac Low M.-M. (2009) Astrophys. J., 704, 1239.

Okuzumi S. (2009) Astrophys. J., 698, 1122.

Okuzumi S. and Hirose S. (2011) Astrophys. J., 742, 65.

Owen J. E. et al. (2010) Mon. Not. R. Astron. Soc., 401, 1415.

Paardekooper S.-J. (2012) Mon. Not. R. Astron. Soc., 421, 3286.

Paardekooper S.-J. et al. (2010) Astrophys. J., 725, 146.

Paardekooper S.-J. et al. (2011) Mon. Not. R. Astron. Soc., 416, L65.

Paczynski B. (1978) Acta Astron., 28, 91.

Pandey B. P. and Wardle M. (2012) Mon. Not. R. Astron. Soc., 423, 222.

Panoglou D. et al. (2012) Astron. Astrophys., 538, A2.

Paoletti M. S. et al. (2012) Astron. Astrophys., 547, A64.

Perez-Becker D. and Chiang E. (2011a) Astrophys. J., 735, 8.

Perez-Becker D. and Chiang E. (2011b) Astrophys. J., 727, 2.

Petersen M. R. et al. (2007a) Astrophys. J., 658, 1236.

Petersen M. R. et al. (2007b) Astrophys. J., 658, 1252.

Pinte C. et al. (2008) Astron. Astrophys., 489, 633.

Pontoppidan K. M. et al. (2010) Astrophys. J., 720, 887.

Raettig N. et al. (2013) Astrophys. J., 765, 115.

Rafikov R. R. (2005) Astrophys. J. Lett., 621, L69.

Rafikov R. R. (2009) Astrophys. J., 704, 281.

Rayleigh L. (1917) Royal Society of London Proceedings Se- 
ries $A, 93,148$.

Regály Z. et al. (2012) Mon. Not. R. Astron. Soc., 419, 1701.

Rice W. K. M. et al. (2011) Mon. Not. R. Astron. Soc., $418,1356$.

Richard D. and Zahn J.-P. (1999) Astron. Astrophys., 347, 734.

Rigliaco E. et al. (2013) Astrophys. J., 772, 60.

Romanova M. M. et al. (2013) Mon. Not. R. Astron. Soc., 430, 699 .

Rüdiger G. et al. (2002) Astron. Astrophys., 391, 781.

Ruge J. P. et al. (2013) Astron. Astrophys., 549, A97.

Ruzmaikina T. V. (1982) Mitteilungen der Astronomischen Gesellschaft Hamburg, 57, 45.

Salmeron R. and Wardle M. (2008) Mon. Not. R. Astron. Soc., 388, 1223.

Salmeron R. et al. (2011) Mon. Not. R. Astron. Soc., 412, 2,1162 .

Salyk C. et al. (2008) Astrophys. J. Lett., 676, L49.

Salyk C. et al. (2011) Astrophys. J., 731, 130.

Sano T. and Inutsuka S.-i. (2001) Astrophys. J. Lett., 561, L179.

Sano T. and Miyama S. M. (1999) Astrophys. J., 515, 776.

Sano T. and Stone J. M. (2002a) Astrophys. J., 570, 314.

Sano T. and Stone J. M. (2002b) Astrophys. J., 577, 534.

Sano T. et al. (2000) Astrophys. J., 543, 486.

Sargent B. A. et al. (2009) Astrophys. J. Suppl., 182, 477.

Schartman E. et al. (2012) Astron. Astrophys., 543, A94.

Schultz-Grunow F. (1959) Z. Angewandte Mathematik und Mechanik, 39, 101.

Semenov D. and Wiebe D. (2011) Astrophys. J. Suppl., 196, 25.

Shakura N. I. and Sunyaev R. A. (1973) Astron. Astrophys., 24, 337.

Shen Y. et al. (2006) Astrophys. J., 653, 513.

Shi J. et al. (2010) Astrophys. J., 708, 1716.

Shlosman I. and Begelman M. C. (1987) Nature, 329, 810.

Simon J. B. and Hawley J. F. (2009) Astrophys. J., 707, 833.

Simon J. B. et al. (2009) Astrophys. J., 690, 974.

Simon J. B. et al. (2011a) Astrophys. J., 730, 94.

Simon J. B. et al. (2011b) Astrophys. J., 743, 17.

Simon J. B. et al. (2012) Mon. Not. R. Astron. Soc., 422, 2685.

Simon J. B. et al. (2013a) Astrophys. J., 764, 66.

Simon J. B. et al. (2013b) Astrophys. J., 775, 73.

Sorathia K. A. et al. (2010) Astrophys. J., 712, 1241.

Sorathia K. A. et al. (2012) Astrophys. J., 749, 189.

Spruit H. C. (1996) in: NATO ASIC Proc. 477: Evolutionary Processes in Binary Stars, (edited by R. A. M. J. Wijers, M. B. Davies, and C. A. Tout), pp. 249-286.

Steiman-Cameron T. Y. et al. (2013) Astrophys. J., 768, 192.

Stone J. M. and Norman M. L. (1992) Astrophys. J. Suppl., $80,753$.

Stone J. M. et al. (2008) Astrophys. J. Suppl., 178, 137.

Suzuki T. K. and Inutsuka S.-i. (2009) Astrophys. J. Lett., 691, L49.

Suzuki T. K. et al. (2010) Astrophys. J., 718, 2, 1289.

Tanga P. et al. (1996) Icarus, 121, 158.

Tassoul J.-L. (2007) Stellar Rotation, Cambridge University Press; 1 edition (July 2, 2007).
Taylor G. I. (1923) Royal Society of London Philosophical Transactions Series A, 223, 289.

Taylor G. I. (1936) Royal Society of London Proceedings Series A, 157, 546.

Terquem C. and Papaloizou J. C. B. (1996) Mon. Not. R. Astron. Soc., 279, 767.

Turner N. J. and Drake J. F. (2009) Astrophys. J., 703, 2152.

Turner N. J. and Sano T. (2008) Astrophys. J. Lett., 679, L131.

Turner N. J. et al. (2006) Astrophys. J., 639, 1218.

Turner N. J. et al. (2007) Astrophys. J., 659, 729.

Turner N. J. et al. (2010) Astrophys. J., 708, 188.

Tzeferacos P. et al. (2009) Mon. Not. R. Astron. Soc., 400, 820.

Tzeferacos P. et al. (2013) Mon. Not. R. Astron. Soc., 428, 3151.

Umebayashi T. and Nakano T. (1981) PASJ, 33, 617.

Umebayashi T. and Nakano T. (1990) MNRAS, 243, 103.

Umebayashi T. and Nakano T. (2009) Astrophys. J., 690, 69.

Umebayashi T. et al. (2013) Astrophys. J., 764, 104.

Uribe A. L. et al. (2011) Astrophys. J., 736, 85.

Urpin V. (2003) Astron. Astrophys., 404, 397.

Urpin V. and Brandenburg A. (1998) Mon. Not. R. Astron. Soc., 294, 399.

van der Marel N. et al. (2013) Science, 340, 1199.

Varnière P. and Tagger M. (2006) Astron. Astrophys., 446, L13.

Velikhov E. (1959) J. Expt. Theor. Phys. (USSR), 36, 1398.

Vorobyov E. I. and Basu S. (2007) Mon. Not. R. Astron. Soc., 381, 1009.

Vorobyov E. I. and Basu S. (2009) Mon. Not. R. Astron. Soc., 393, 822.

Vorobyov E. I. and Basu S. (2010) Astrophys. J., 719, 1896.

Wardle M. (1999) Mon. Not. R. Astron. Soc., 307, 849.

Wardle M. (2007) Astrophys. Space Sci., 311, 35.

Wardle M. and Königl A. (1993) Astrophys. J., 410, 218.

Wardle M. and Salmeron R. (2012) Mon. Not. R. Astron. Soc., 422, 2737.

Watson A. M. et al. (2007) Protostars and Planets V, pp. $523-538$.

Weidenschilling S. J. and Cuzzi J. N. (1993) in: Protostars and Planets III, (edited by E. H. Levy and J. I. Lunine), pp. 1031-1060.

Weiss B. P. et al. (2010) Space Sci. Rev., 152, 341.

Wendt F. (1959) Ingenieur-Archiv, 4, 557.

Willacy K. et al. (2006) Astrophys. J., 644, 1202.

Wolf S. and Klahr H. (2002) Astrophys. J. Lett., 578, L79.

Zanni C. et al. (2007) Astron. Astrophys., 469, 811.

Ziegler U. (2005) Astron. Astrophys., 435, 385.

Ziegler U. (2008) Computer Physics Communications, 179, 227.

Zolensky M. E. et al. (2006) Science, 314, 1735.

Zsom A. et al. (2010) Astron. Astrophys., 513, A57+.

This 2-column preprint was prepared with the AAS LATEX macros v5.2. 